\title{
Formal Analysis of Log Files
}

\author{
Howard Barringer* \\ University of Manchester, Manchester, UK \\ Alex Groce ${ }^{\dagger}$ \\ Oregon State University, Corvallis, Oregon \\ and \\ Klaus Havelund ${ }^{\ddagger}$ and Margaret Smith ${ }^{\S}$ \\ Jet Propulsion Laboratory, California Institute of Technology, Pasadena
}

DOI: $10.2514 / 1.49356$

\begin{abstract}
Runtime verification as a field faces several challenges. One key challenge is how to keep the overheads associated with its application low. This is especially important in real-time critical embedded applications, where memory and CPU resources are limited. Another challenge is that of devising expressive and yet user-friendly specification languages that can attract software engineers. In this paper, it is shown that for many systems, in-place logging provides a satisfactory basis for postmortem "runtime" verification of logs, where the overhead is already included in system design. Although this approach prevents an online reaction to detected errors, possible with traditional runtime verification, it provides a powerful tool for test automation and debugging —in this case, analysis of spacecraft telemetry by ground operations teams at NASA's Jet Propulsion Laboratory. The second challenge is addressed in the presented work through a temporal specification language, designed in collaboration with Jet Propulsion Laboratory test engineers. The specification language allows for descriptions of relationships between data-rich events (records) common in logs, and is translated into a form of automata supporting data-parameterized states. The automaton language is inspired by the rule-based language of the RULER runtime verification system. A case study is presented illustrating the use of our LOGSCOPE tool by software test engineers for the 2011 Mars Science Laboratory mission.
\end{abstract}

\section{Introduction}

$\mathrm{R}$ UNTIME verification encompasses the discipline of checking execution traces against formal specifications. Two of the key problems are: 1) how to obtain execution traces with minimal impact on observed software, and without over-burdening the user with the need to implement instrumentation and 2) how to design a specification language that is expressive, yet user-friendly. This paper reports on an experiment addressing both these issues, a combination that we call "low-impact" runtime verification. In summary, the paper argues that analyzing log information removes the instrumentation burden in the sense that logs usually are already produced by most critical software systems.

Received 13 February 2010; accepted for publication 10 May 2010. Copyright $\odot 2010$ by the American Institute of Aeronautics and Astronautics, Inc. The U.S. Government has a royalty-free license to exercise all rights under the copyright claimed herein for Governmental purposes. All other rights are reserved by the copyright owner. Copies of this paper may be made for personal or internal use, on condition that the copier pay the $\$ 10.00$ per-copy fee to the Copyright Clearance Center, Inc., 222 Rosewood Drive, Danvers, MA 01923; include the code 1542-9423/10 \$10.00 in correspondence with the CCC.

* School of Computer Science, howard.barringer@manchester.ac.uk.

$\dagger$ School of Electrical Engineering and Computer Science, alex@eecs.oregonstate.edu

¥ Laboratory for Reliable Software, klaus.havelund@jpl.nasa.gov

$\S$ Software System Engineering, margaret.h.smith@jpl.nasa.gov 


\section{BARRINGER ET AL.}

Consequently, no additional impact on the running software is imposed beyond the already implemented logging. A $\log$ is a recorded sequence of events. We argue that systematizing logging can be of additional benefit. Second, we introduce a textual temporal logic-inspired specification language over events for specifying properties of logs. This language supports data-parameterization, essential for monitoring logs (events typically carry data). Specified properties, called patterns, are translated into data-parameterized automata forming an interesting and useful subset of the textual RULER language [1-4]. Users can mix patterns with more expressive automata in a specification. Parameterized automata are visualized using the GRAPHVIZ tool [5]. Our system additionally offers preliminary support for automatically constructing (learning) specifications from example logs. The log analysis framework, LOGSCOPE, is developed to support engineers testing the flight software for NASA's next Mars rover mission, the Mars Science Laboratory (MSL) [6], developed at the Jet Propulsion Laboratory (JPL). In this sense the work represents an instance of the often sought marriage between theory and practice in formal methods.

The MSL mission's goal is to put the so-far largest rover (the size of a compact car) on Mars for continued exploration of the red planet. It is scheduled for launch in 2011. A flight software team peaking at approximately 30 programmers develops the software for controlling the rover compute element, which controls all stages of the integrated spacecraft. A testing team of approximately 10 engineers (the Flight software Internal Test, or FIT, team) is responsible for functional testing of the flight software. LOGSCOPE was developed to support this team, and is the result of their requirements as well as our research ideas. The MSL flight software produces rich log information, which is stored in SQL databases (one database per $\log$ ). A log is in essence a sequence of time-stamped events, where an event can be one of several forms, corresponding to input to and output from the system, as well as internal state transitions and state readings. Each event is in essence a mapping from field names to values (a record). Such logs are usually far too large to be analyzed effectively by humans. Traditionally, these MSL logs have been analyzed by writing scripts, with properties coded up in PYTHON. Such scripts are time consuming to produce and result in difficult-to-read "specifications" that hinder communication, maintenance, specification-sharing, and reuse. Based on our experience with LOGSCOPE, runtime verification of logs using formal specifications has excellent potential as a route to introduce lightweight formal methods into a high-profile NASA flight project.

\section{A. Contributions and Related Work}

The contribution of this work is the design of a simple, user-friendly, and yet for practical purposes sufficiently expressive data-parameterized temporal specification language, with a translation into data-parameterized automata, which are themselves usable for specification. A second contribution is the injection of this technology into a NASA flight mission. The LOGSCOPE system has specifically been influenced by the RULER system [1-4]. In particular, the automaton language conceptually forms a subset of RULER. LOGSCOPE can be seen as an adaptation of RULER to the specific needs of MSL: adding temporal logic, enhancing usability, and implementing it in PYTHON to integrate with the existing scripting culture. LOGSCOPE has also been influenced by the state machine-based systems RCAT [7] and RMOR [8]. The relationship between LOGSCOPE and RULER is illustrated in [1], whereas the process that led to LOGSCOPE is presented in [9].

Within the last decade, a substantial body of work has been produced on runtime verification [10], e.g., to mention just a few approaches, over and above the work referenced above, consider [11-21]. Each of these is characterized by several dimensions, such as the specification logic they support, the way in which they connect with the application being monitored, the way in which monitors are generated for the propositional (data-free) case, and the way in which they handle data values. LOGSCOPE offers a user-friendly logic, avoiding some of the pitfalls of LTL (such as the need for nested until-formulas for expressing sequencing). By analyzing log files, the issue of code instrumentation becomes a separate problem. Data parameterization is handled through parameterized automata: a state machine state can carry a vector of data values. The approach is a restricted form of parameterization mechanisms in the RULER system (see Sec. V.D), however, the basic idea is a special case of the monitor state being a formula including data. It is also used in our own EAGLE system [12], which offers a very sophisticated logic based on minimal and maximal fixpoints. The sophistication of this logic caused the implementation to be highly complex. The HAwK system [14] augmented EAGLE with an event definition language specialized for Java, and monitors were generated as ASPECTJ [22] aspects. JLO [21,23] generates monitors in a similar way from parameterized LTL formulas. As explained in [23], the monitor state is a formula including instantiated data values. For example, consider the LTL formula: $\square(p(x) \Rightarrow \diamond q(x)$ ) ("always: when $p(x)$ is observed, where $x$ is a binding variable 


\section{BARRINGER ET AL}

name, then eventually $q(x)$ must be observed"). This formula, upon an observed event $p(1)$, will be rewritten as $\square(p(x) \Rightarrow \diamond q(x)) \wedge \diamond q(1)$. JLO events are defined by pointcuts inspired by aspect-oriented programming, and monitors are generated as ASPECTJ aspects.

Often the generality of parameterization mechanisms is sacrificed for optimality. An example is the TRACEMATCHES system [11], which carries the connection to ASPECTJ to the extreme and extends ASPECTJ with regular expressions. Data values are handled by annotating state machine states with constraints, and updating these as events are observed. Chen and Roşu [13] implement a range of traditional logics, such as state machines, temporal logic, regular expressions, and grammars, as different plugins in the MoP system. Data (parameters) are handled uniformly in Mop by separating them out from the logic plugins, which are all propositional. Monitors are instead indexed by data values. This makes designing a new logic very easy since only the propositional case needs to be defined. It also makes monitoring very efficient. Events are generated by definition of pointcuts, and monitors are generated as ASPECTJ aspects. As already alluded to, the logics supported by these different systems have drawbacks. For example, state machines are verbose (one has to mention intermediate states), and LTL as well as regular expressions can be difficult to use.

Software testing researchers [24,25] have also explored formal specifications for checking logs. Chang and Ren [25] provide a specification language just based on regular expressions. The properties are formulated over actual textual logs, in contrast to LOGSCOPE properties, which are formulated over an abstraction of the log: a sequence of records, each of which is a mapping from field names to values. This makes their approach less adaptable to changes in the log format. Chang and Ren provide interesting empirical data on application to a large telecommunications system (though it is not clear whether specifications are written by the testers or the research team). Andrews and Zhang [24] share our focus on lightweight test oracles. They offer an elegant parameterized state machine framework similar to LOGSCOPE parameterized state machines. Their state machines yield errors in case events occur for which there are no outgoing transitions. Although we support this concept, we also support state machines where one can wait in a state until a certain event occurs. This has turned out to be useful in practice for large log files and occasionally leads to less verbose specifications in the case studies we have looked at. Their state machines are compiled into PROLOG, preventing an online application of monitoring in case this should be desired. They do not provide a temporal logic, a concept that we found important for acceptance by engineers.

The PsL specification language [26] combines temporal logic and regular expressions, and in this way has commonalities with the LOGSCOPE language. However, PSL is designed for hardware verification and does not support data parameterization to the degree that LOGSCOPE does. The LOGSCOPE temporal specification language is characterized by integrating temporal logic and a limited form of regular expressions in a very simple manner (there are, for example, no explicit temporal operators). The LOGSCOPE specification language is much simpler than PsL. Engineers were able to write properties in less than one hour. Finally, LOGSCOPE offers parameterized state machines, whereas this concept is not directly supported in PSL. Like PSL and LOGSCOPE, the SALT specification language [27] has been developed to make specification writing easier for industrial use. SALT is an impressive collection of temporal operators, which are translated to LTL, or timed LTL in case time constraints are involved. SALT offers operators similar to LOGSCOPE's sequencing operator and scope operator (upto, which in SALT is rejecton and accepton). The goals of PSL and SALT are important. The distinctive features of LOGSCOPE is its simplicity compared with those languages, although still attempting to improve the usability of LTL, and the fact that LOGSCOPE handles data parameters over very large (or infinite) data domains, which is not the case for either PSL or SALT.

Mateescu and Thivolle [28] introduce a model checking language (MCL), which augments the modal $\mu$-calculus with high-level operators aimed at improving expressiveness and conciseness of formulas. The main MCL ingredients are data-parameterized fixed points, action patterns extracting data values from events, modalities on transition sequences described using extended regular expressions and programming language constructs. The attempt in [28] is similar to the work described in [12] on extending the $\mu$-calculus with data and a sequencing (chop) operator for runtime monitoring. However, although very expressive we do not believe that formulas in McL are necessarily easy to write for engineers. Our framework is simpler and directly applicable to formulate the kinds of properties needed for our case studies.

Coen-Porisini et al. [29] introduce the PDL specification language for stating properties about programs, to be verified using symbolic execution. The specification language has some similarities with our pattern language in providing a form of sequencing operator and allowing for negation of events. However, PDL is limited in the alternation 
between positive and negative events allowed. More important, events can only be program labels. There is no support for data parameterization.

Dillon et al. introduce in [30] the Graphical Interval Logic (GIL) for specifying the behavior of concurrent systems. The logic is quite powerful and addresses the problem of usability of propositional temporal logic. However, the GIL logic is considerably more complex than the logic presented here, making fast learning by test engineers less likely. The LOGSCOPE language is textual as opposed to GIL, which is a graphical notation. It has been our experience that test engineers work faster with a good textual notation rather than a graphical notation. It also makes it easier to auto-generate specifications. Finally, GIL does support some form of data quantification. However, there are no algorithms provided for monitoring such unless the type quantified over is finite and known [31].

The work in [32] describes a set of specification patterns often encountered in work on verification. Each pattern focuses on one specific property format, such as, for example, the response pattern ( $P$ should always be followed by $Q$ ). A central part of these specification patterns are scopes, which LoGSCOPE to some degree has implemented in terms of the upto construct. Our experience is that our general language is simple enough for engineers to work with, and that special patterns are not necessarily needed. This is in fact the strength of the proposed language in our belief. The specification patterns in [32] do not deal with data, and it would likely be a research topic to extend the patterns with such.

\section{B. Outline of Paper}

Section II describes the process that lead to the design of the LOGSCOPE specification language and system. Section III describes how log files are constructed for the MSL flight software, forming the foundation of a through-going example. Section IV introduces the temporal pattern language, whereas Sec. V introduces the automaton language and illustrates how patterns are translated to automata. Section VI briefly describes the learning feature. Section VII describes the results of the MSL case study in more detail. Section VIII finally concludes the paper and suggests future work. An appendix contains the complete grammar for the LOGSCOPE specification language.

\section{Tool Development Process}

The LOGSCOPE tool and pattern language grew out of the needs of engineers. In fact, the earliest effort to "mockup" a specification came from an MSL test engineer. The "specification" was a comment in a PYTHON script describing the purpose of the script as follows:

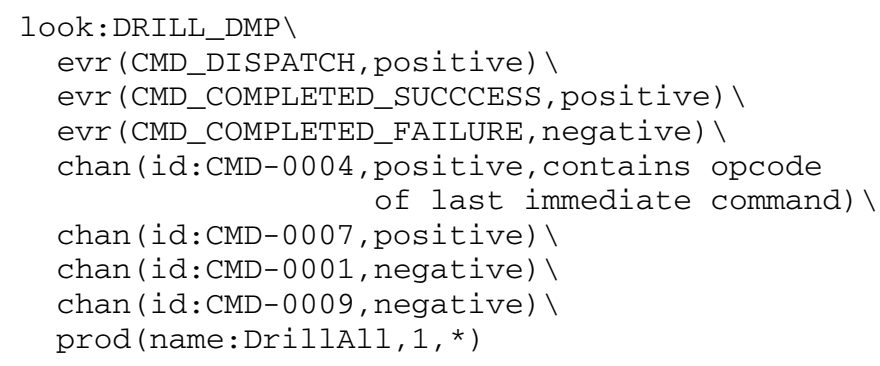

The script represents the following property to be checked: when an issued DRILL_DMP command is observed in the log, then the events with positive as an argument should follow in any order, and the events with negative as an argument should not occur. For example, the following evr (event report) events should follow: a report of the dispatch (CMD_DISPATCH) of the command; a report of the success (CMD_COMPLETED_SUCCCESS) of the command; and there should not be an evr reporting failure (CMD_COMPLETED_FAILURE) of the command. Then follows some requirements on samplings of the state (channel events). For example, there should be a sampling of state variable CMD-0004 that, informally stated, contains the opcode of the last immediate command. Finally, the flight software should downlink a DrillAll product (a prod event) to ground informing about the status of the drill. "To downlink" is NASA terminology for sending information from spacecraft to ground. 


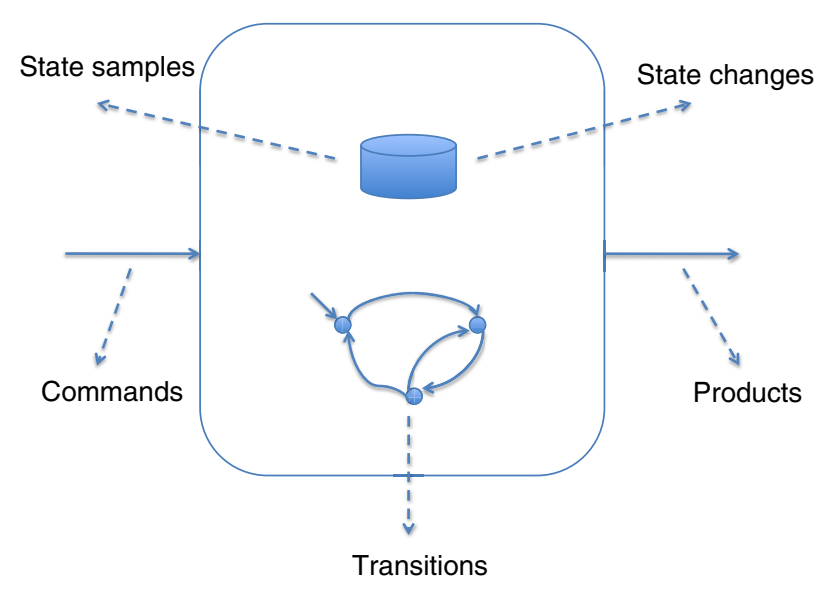

Fig. 1 Observable events of a system.

The core elements of our temporal pattern language - the events, all originate in the telemetry system of the MSL flight and ground software. These events correspond to the generalized view of a "system" shown in Fig. 1. An operations team on the ground issues commands to the spacecraft, e.g., DRILL_DMP in the sample specification. The spacecraft software responds to commands by causing transitions. Transitions in turn cause the local state to be updated. State changes can be observed either in terms of "random" state samples that provide a snapshot of current spacecraft state, or more precisely by observing the actual state changes when they occur. Finally, data products are the "outputs" of the spacecraft downlinked to the ground, including engineering telemetry, images, and science instrument data. These different event kinds are referred to in the logs (see Fig. 2) as, respectively: COMMAND, EVR (EVent Report $=$ transition), CHANNEL (state sampling), CHANGE (state change), and PRODUCT. Although these MSL concepts are very general (should cover any system) LOGSCOPE can be adjusted to work with other forms of events.

To a large extent, the development of the pattern language that followed was a process of taking this early draft and preserving its essential elements while expanding its expressive power and readability, guided by usage, engineer requests, and language design considerations. For example, we preserved the structure of properties, each having the form: $E \Rightarrow C$, where $E$ is an event and $C$ is a consequence, for example, a sequence of events. However, more complex specifications were required. In the original mockup, the events specified positive in $C$ were allowed to occur in any order in the log. The first implementation prototype introduced a way to distinguish ordered lists from unordered sets of events, which proved critical to real specifications.

The original test infrastructure, as suggested by the FIT team, provided an event registry, which was a PYTHON library with methods allowing test scripts to create, alter, and delete online monitors for telemetry events. In principle, the registry provided a set of tools sufficient for analyzing tests at runtime, and a specification and debugging mechanism well-integrated with established testing procedures. In practice, the registry approach was seldom adopted by engineers (and proved frustrating when used) for the following two primary reasons:

1) Ordering and timing of events: Events on the spacecraft are not downlinked to the ground system in chronological order. Different channels, data products, and EVRs have different priorities, and the "Earth receive time" ordering of two events will often be the opposite of their event times. Test engineers were forced to either introduce lengthy delays after each test step or build very complicated logic to disentangle events arriving out-of-order and "recreate" a linear chronology of events (which is sometimes only possible after test termination). A strategy of pausing long enough to allow telemetry to arrive proved brittle, as timeouts fluctuated with each software release.

2) Confusion of test execution and test evaluation: A motivation for the event registry was that it seamlessly integrated with the idea of tests as scripts with logic, looping, and other programming language features. Unfortunately, test scripts that were clearly readable when their task was limited to commanding the spacecraft became difficult to follow when test execution was interlaced with test evaluation. 
BARRINGER ET AL.

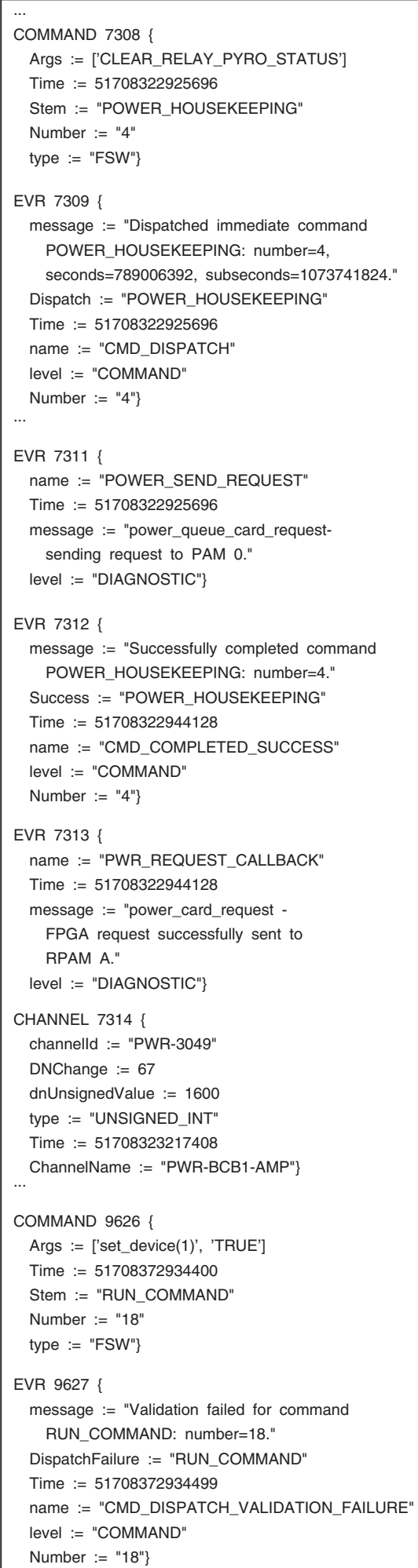

Fig. 2 A simplified log. 
Neither of these problems is particular to MSL. The first problem is generic to any distributed system in which constructing an event timeline is nontrivial (before queues clear), and the second problem is a general observation about test case readability. Test engineers reacted to these problems by making little use of the registry, despite having requested its features. How, then, were test engineers evaluating tests? In many cases, they were hand-scripting a post-test analysis of all telemetry (ad hoc construction of very limited logs). A few checks were routinely performed online, but more complex analysis was often delayed until the test was complete, and chronological confusion could be partially avoided by counting expected responses.

Team management (also active in writing tests) recognized that building ad hoc test-specific "log analysis" systems was not an effective use of mission time, and that scripting logic only partially addressed both problems with the registry. The DRILL_DMP mockup emerged after a series of team meetings and discussions, informed by our ideas of $\log$ analysis. MSL flight software management suggested that a unified logging scheme would be useful to developers outside the test team. After an initial trial run in which the research team replicated the hand-scripted results of random command regressions, the FIT team began use of prototype versions of the logging system, replacing hand-scripted test evaluation code, as described in more detail in Sec. VII. The research team suggested the use of square and curly braces to indicate lists and sets of events, respectively, and the use of bindings for data values. Other features, including indexed data fields (e.g., bit-vectors), SQL query filtering of events, scoping of specifications (Sec. IV.F), and learning (Sec. VI), were requested by the test team. A specification language emerged, starting with a syntax and informal semantics inspired by the original mockup, powerful enough to support test needs, and simple enough to actually use.

\section{Logs}

The first step in analyzing the behavior of the MSL flight system is to produce a log — an ordered sequence of events. A $\log$ is extracted from a database of spacecraft telemetry maintained by JPL ground software. The ground software will be used by mission operations to communicate with the spacecraft and rover, and is used both in MSL hardware testbeds and software simulation. The ground system stores events of each system execution as entries in an SQL database specific for that execution (an approach also considered in runtime verification literature [33]).

The log extracted from such a database is simply an ordered list of named records (effectively a sequence of dictionaries in PYTHON). All MSL-specific aspects of the log are handled by the log extractor tool (LOGMAKER), making LOGSCOPE easily adaptable to any system producing logs: one just has to replace the log extractor. Different kinds of events in the database provide different fields (e.g., EVRs have a message but no value, whereas channels have a value but no message). Rather than forcing a single representation on these event types, we allow different events in the extracted $\log$ to have varying fields, but ensure that each event has 1) a type and 2) a timestamp. The type is used in specifications as shown below, and the timestamp allows us to order the event sequence.

Figure 2 shows a simplified print of a log by our tool after testing of the power subsystem. Details have been modified in order to not reveal the exact nature of the log. The example includes COMMAND, EVR, and CHANNEL events. The fields of the events are shown within curly brackets, except for the object type, which appears before the bracket. In addition to fields present in the original database, our system annotates events with derived fields that ease readability and specification. A derived field is computed from fields in the data base, yielding additional useful information. Field names from the MSL database begin in lowercase, whereas derived fields (e.g., Dispatch) begin in uppercase. The Time field, used to order events, is always derived. Events that take place on the spacecraft include a spacecraft event time (omitted here) that establishes a canonical order. However, command events originate from the ground, and include only a transmission time. We establish a uniform chronology by extracting the time a command is dispatched on the spacecraft from the time information in the message of the subsequent dispatch EVR event-the Time field of COMMAND 7308 is extracted from the message in EVR 7309. Use of our tool by test engineers has increased the MSL software team's awareness of ambiguities in the timing of events originating on the spacecraft, suggesting that improved synchronization is needed between modules responsible for different types of telemetry.

\section{Pattern Language}

Monitors are written in the LOGSCOPE specification language. A specification consists of one or more specification units, each of which is either a temporal logic pattern, or a parameterized automaton: 
BARRINGER ET AL.

$$
\begin{gathered}
\langle\text { specification }\rangle \rightarrow\langle\text { monitorspec }\rangle^{+} \\
\langle\text {monitorspec }\rangle \rightarrow\langle\text { pattern }\rangle \mid\langle\text { automaton }\rangle
\end{gathered}
$$

In this section, we focus on temporal patterns, which are automatically translated into parameterized automata.

\section{A. Simple Response Patterns}

Consider as an example the following informally stated property:

$R_{1}$ : "Whenever a flight software command is issued, then eventually an EVR should indicate success of that command".

Before we can formalize this property, it needs to be refined to refer to the specific fields of events. The following is such a refinement:

$R_{1}^{\prime}$ : "Whenever a COMMAND is issued with the Type field having the value "FSW", the Stem field (command name) having some unknown value $x$, and the Number field having some unknown value $y$, then eventually an EVR should occur, with the field Success mapped to $x$ and the Number field mapped to $y$ ".

The Number field is a sequence number indicating the order in which commands are received by the spacecraft for dispatch. Subsequent commands should have increasing numbers. Events related to command execution have the number of the command. In our language, this property reads:

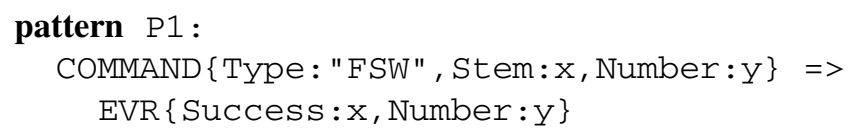

This pattern (pattern is a keyword) has the name P1 and states that if a command is observed in the log at a position $i$, with the Type field having the exact string value "FSW", the Stem field having some value $\mathrm{x}$, and the Number field having some value $\mathrm{y}$, then later in that $\log$, at a position $j>i$, an EVR should occur with a Success field having $\mathrm{x}$ as value and a Number field having $\mathrm{y}$ as value. Informally, we can explain the semantics of this formula in terms of the following formula in an LTL [34] with quantification over data variables:

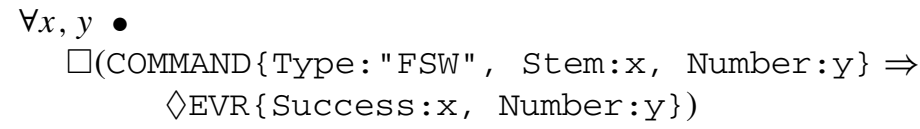

The temporal operators $\square$ and $\diamond$, as well as the universal quantification $\forall$, are implicit in LOGSCOPE's pattern notation. The pattern has the following form:

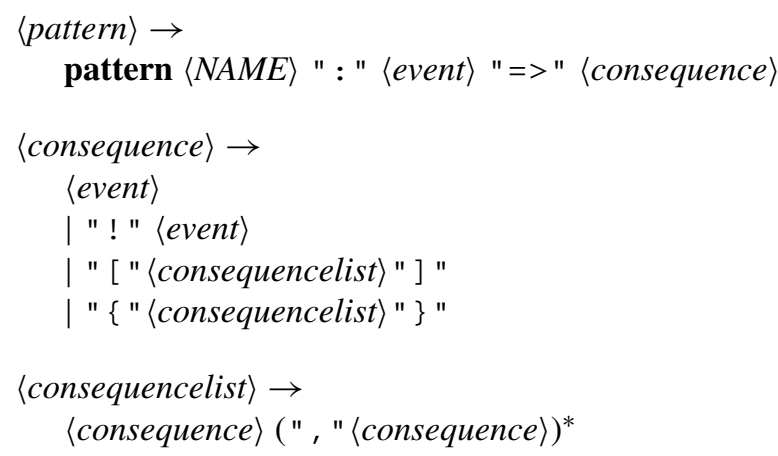

This example shows the simplest case, where the consequence is an event. The other alternatives will be explained in the following subsections. The event triggering the pattern is a command event here, but can be any kind of event. 
Each event is constrained (between $\{\ldots\}$ brackets) by zero or more constraints, each consisting of a field name (without quotes) and a range specification. We saw two forms of range specifications: the string "FSW" for the field Type and the names $\mathrm{x}$ and $\mathrm{y}$ for the other fields. A string constant represents a concrete constraint: the field in the event has to match this value exactly (by PYTHON equality ==). One can also provide an integer as such a concrete range constraint. Ranges can also be multivalued, such as integer intervals, as will be shown below.

An unquoted name ( $\mathrm{x}$ and $\mathrm{y}$ in this case) occurring as a range indicates, at specification time, an unknown value. The first occurrence of such a name in a pattern is binding: it will be bound to the value of the corresponding field in the matching event. Any subsequent occurrences of this name in the pattern are now constraining: for a match to occur, the corresponding fields now should have the values these names were bound to by the binding event. The log in Fig. 2 satisfies this specification for the first command, as the command is matched by a success. The property fails for the second command.

\section{B. Negation of Events}

A consequence can also be the negation (“"”) of an event. Suppose we want to state the following property:

$R_{2}$ : "Whenever a COMMAND is issued with the Type field having the value "FSW", the Stem field having some value $x$, and the Number field having some value y, then there should thereafter not occur an EVR, with the field Failure mapped to $x$ and the Number field mapped to $y "$.

We express this by the property (which the log from Sec. III satisfies):

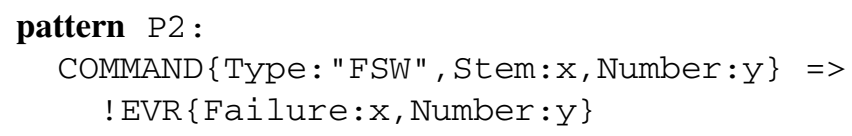

\section{Composite Consequences}

We have seen that the consequence of a pattern can be an event, as in pattern P1, or the negation of an event, as in pattern P2. There are two more forms: ordered and unordered sequences of consequences (a recursive definition). The square brackets [ . . ] indicate that the consequences should occur in exact order, whereas the curly brackets $\{\ldots\}$ indicate that they may occur in any order. The symbols are chosen for their frequent use in literature to represent ordered lists and unordered sets. As an example, consider the following requirement:

$R_{3}$ : "Whenever a flight software command is issued, there should follow a dispatch of that command, and then exactly one success of that command. Before the dispatch there should be no dispatch failure, and in between the dispatch and the success there should not be a failure".

Requirement $R_{3}$ is in Fig. 3 illustrated as a timeline, much like in TIMEEDIT [35], showing events that could/should occur, and constraints on events that should not occur. Note, however, that TIMEEDIT does not support data parameterization, only temporal ordering of atomic events. The property can be stated formally as follows, in a form that very closely reflects the timeline.

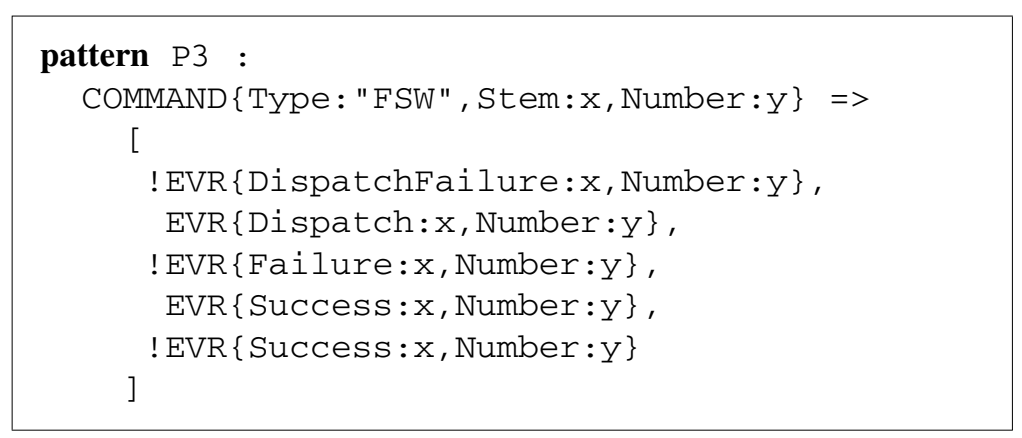




\section{BARRINGER ET AL.}

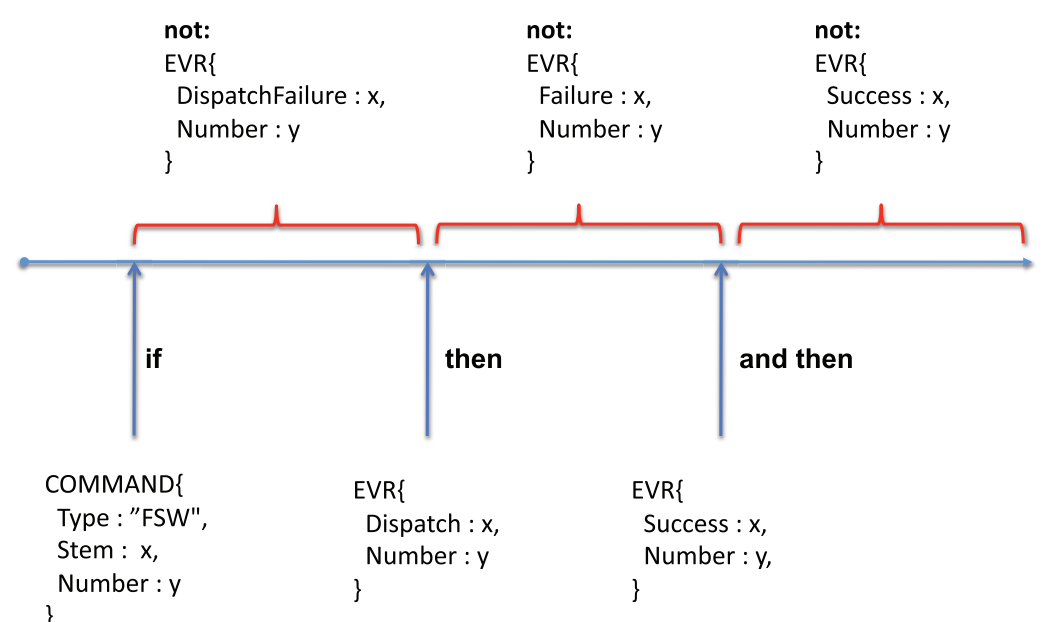

Fig. 3 Requirement $R_{3}$ expressed as a timeline.

The consequence consists of a sequence (in square brackets [ . . ] ) of (sub) consequences, in this case events and negations of events. The ordering means that as a response to the command the dispatch should occur before the success, and the negations represent what should not happen in between the nonnegated events.

The following first-order LTL formula represents this property. It is suggestive of the extra complication that LTL's until operator (" $\mathcal{U}$ ") can introduce, and why the pattern language might be easier to use for engineers:

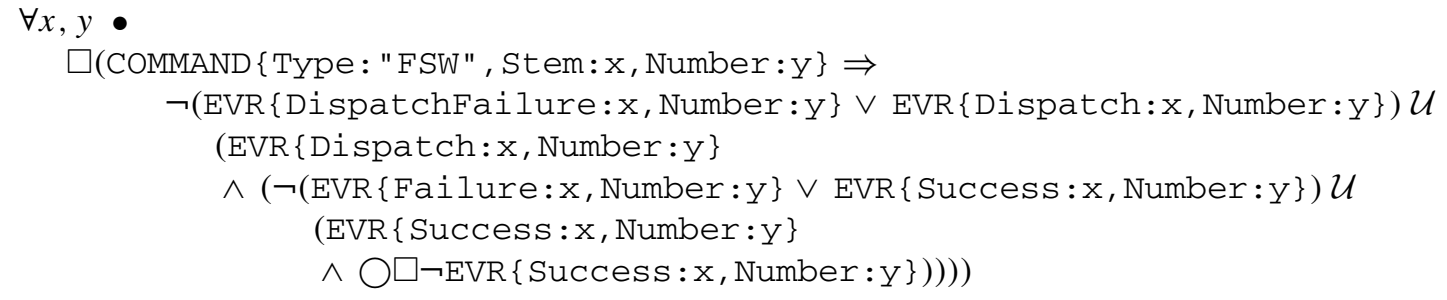

Linear time temporal logics have by several authors been extended with chop operators ("; "), for example, [36]. This eliminates the nesting problem of the until operator, although a recoding of the given example (and adding quantification over data variables) is still nontrivial:

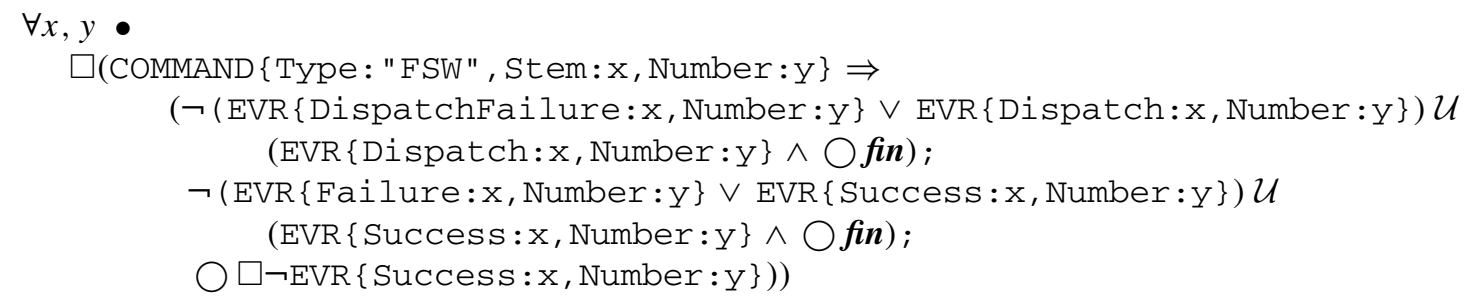

The fin predicate is true on the empty trace. The chop (in [36]) is overlapping, and hence the need to apply the $\bigcirc$ operator to the final $\square$ term. As an example of an unordered arrangement of events, consider the following (perhaps less meaningful) relaxation of the above stated property:

$R_{4}$ : "Whenever a flight software command is issued, there should follow a dispatch of that command and exactly one success of that command, in no particular required order. There should be no dispatch failure and no failure at any time". 
We formalize this as follows:

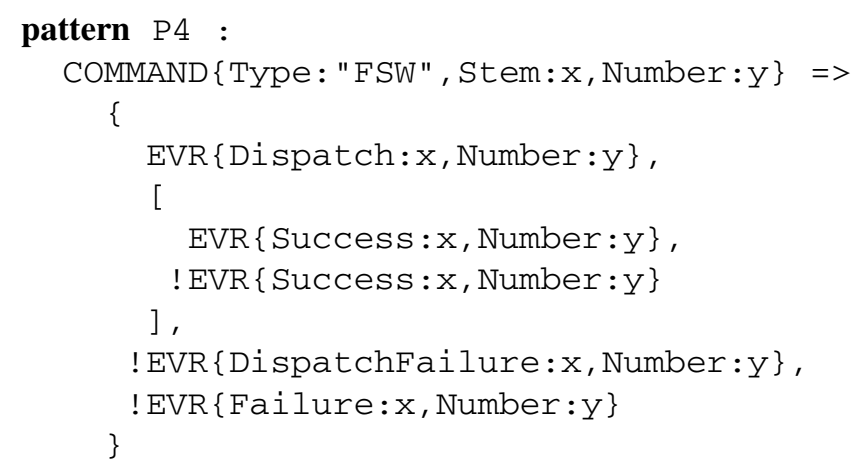

The curly brackets $\{\ldots\}$ indicate an unordered collection of consequences (a Boolean “ $\wedge$ ” effectively). This corresponds in this case to four parallel timelines, all of which must be satisfied. The fact that they are unordered means that the non-negated events can occur in any order (but must occur eventually), and negations have to hold to the end of the $\log$. Nested inside the unordered $\{\ldots\}$ construct there is, however, an ordered sequence expressing that after a success there should not occur another success. As the grammar for consequences suggests, ordered and unordered collections of consequences can be mixed arbitrarily.

\section{Event Predicates}

Events can be constrained with predicates. Consider the following requirement:

$R_{5}$ : "The success of a command with a number y should never be followed by the success of a command with an equal or lower number $z \leqslant y$ ”.

The following pattern formalizes this requirement.

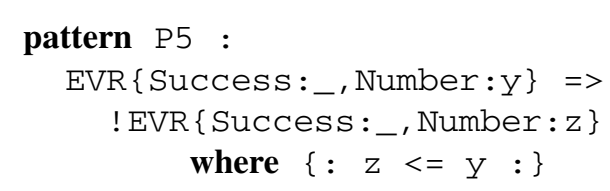

Note that this formula ignores which commands succeed (the underscore "_"). The constraining predicate is: $\{: \quad z<=y:\}$ and is a general Python expression enclosed by the symbols $\{: \ldots:\}$. This expression can refer to all of PyTHON's directly available features, in addition to a LOGSCOPE-specific predefined library of predicates. Should this not suffice, it is possible to import PYTHON libraries or directly define PYTHON predicates in the specification. The following specification defines a PYTHON predicate within $(t 1, t 2, \max )$, which checks that the two time points $t 1$ and $t 2$ are at most max time units apart.

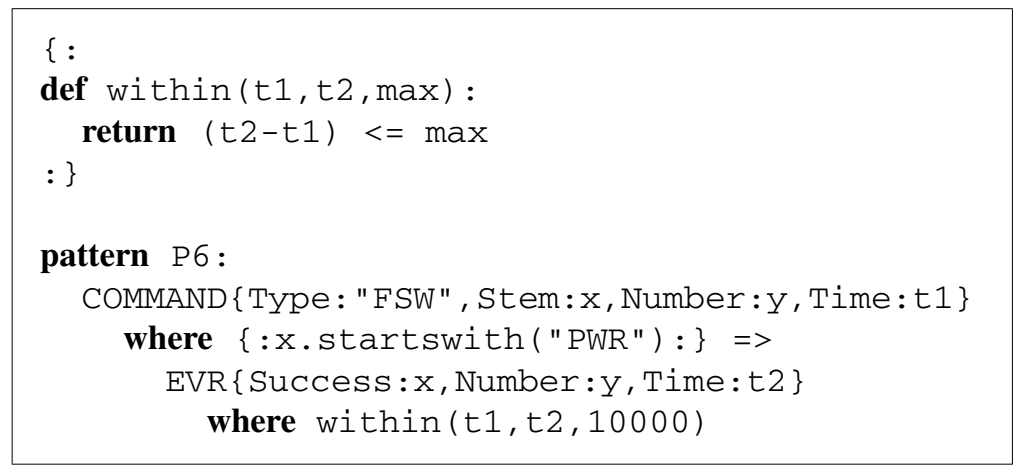


PyTHON code must occur at the beginning of the specification file and be delimited by the symbols $\{: \ldots:\}$. This predicate is then used to check that power commands (having names starting with " PWR") succeed within maximally 10,000 time units. The $\mathrm{x}$. startswith (y) method is a PYTHON built-in, returning true if the string $\mathrm{y}$ is a prefix of the string $x$. Note that it is possible to call a predicate directly without embedding it within the more general code brackets $\{: \ldots:\}$. Predicates can be composed using the traditional Boolean operators: or, and, not.

As a matter of convenience there are shorthands for certain predicates that occurred frequently during testing of the MSL flight software, such as interval checks and indexing operations, in particular bit-indexing in bit-vectors. The following example illustrates this:

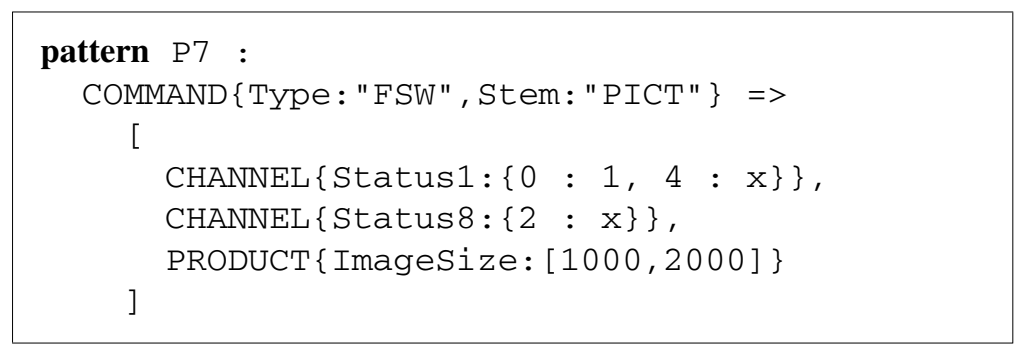

The requirement states that after a flight software picture command, there should be a channel reading where the Status 1 field denotes an integer, where bit number 0 is 1 (counted from the right), and where bit 4 is bound to some value $\mathrm{x}(0$ or 1$)$. Thereafter, another channel reading should follow where the Status 8 field denotes an integer, where bit 2 has the value $x$. Finally a product, should follow, where the field ImageSize is in the interval 1000-2000. The form of indexing shown for the status fields (Status1 and Status8) generally works on any data object that is indexable, such as bit-vectors (integers), lists and strings (in both latter cases indexes must be integers, counting from the left), and dictionaries (maps, allowing for indexing also with strings).

\section{E. Event Actions}

As a more experimental feature (not used by the test engineers), LOGSCOPE allows events to be associated with actions to be executed when the events match. As an example, consider the following specification, which is a weakening of property P1. It states that only the first command must be followed by a success EVR.

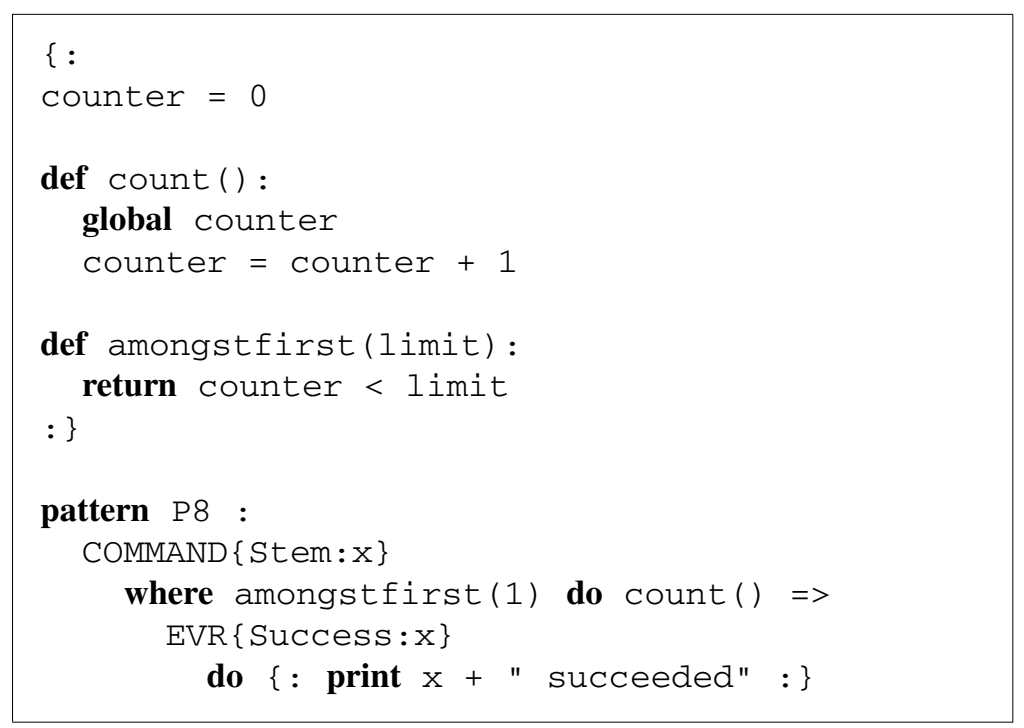


The specification first introduces a PYTHON code section: a global integer counter, a count ( ) function, and an amongstfirst (limit) function. If a command still matches the where predicate (it is among the first 1 commands), the do construct is executed, causing the count ( ) function to be called, counting up the global counter variable. Just as predicates can be referred to by name (without enclosing in $\{: \ldots:\}$ ), so can actions as in this example. The general format of an event is as follows:

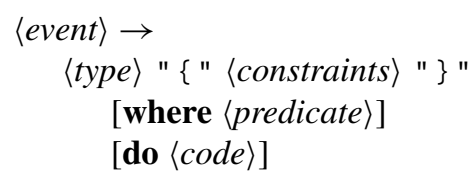

If the user-defined PyTHON code contains the definition of a method final ( ) (without aguments), then this method will be called at the end of monitoring the properties. This function can, for example, compute and print statistics based on the state of the global variables introduced. Each specification file has its own Python state. Hence, it is possible to declare a state local to a subset of properties.

\section{F. Scopes}

In some cases one may want to limit the scope in which a pattern is checked, by providing an additional scopeterminating event. Without such limitations a pattern holds from the point at which its trigger event is matched until the end of the log. Scopes were introduced in the specification patterns described in [32] and also exist, for example, in SALT [27] (specifically what is referred to there as exceptions: rejecton and accepton). As an example, one may want to check that a particular command results in a particular set of events to occur, and some other events not to occur, up to the next command being fired. Consider, for example, pattern P4 in Sec. VI.C. According to the semantics, whenever a flight software command is detected in the log, the consequence is checked on the rest of the $\log$, to its end; that is, any required event such as the dispatch can occur anywhere in the rest of the log, and negative events, such as failures, are checked on the remaining log. We might, however, limit these checks to be performed up to the next flight software command (satisfying COMMAND \{Type: "FSW" \}). This is done by adding the scope delimiter upto COMMAND \{ Type: " FSW" $\}$ as follows:

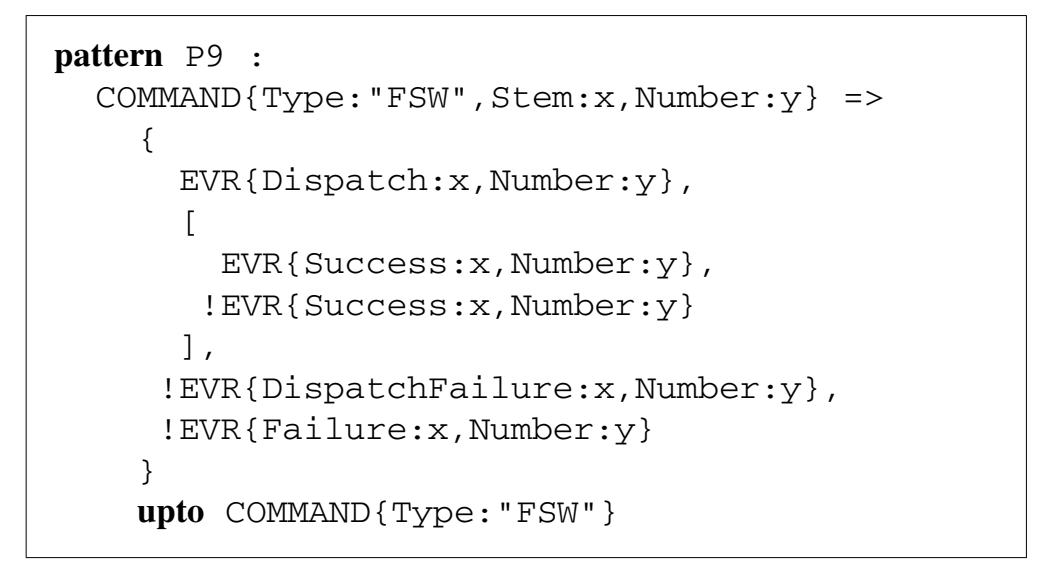

This means that positive events such as the dispatch have to occur before the next flight software command, and negative events, such as failures, are only checked for (forbidden) up to the next flight software command. The syntax for patterns is now expanded to include an optional scope-specification:

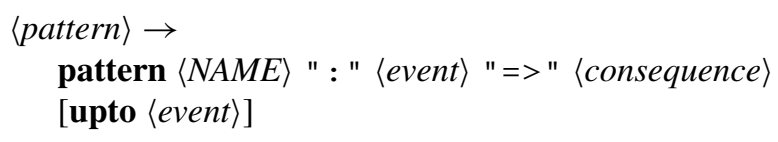




\section{Automaton Language}

LOGSCOPE also allows testers to write properties as parameterized finite-word $\forall$-automata, a more expressive, but lower-level language than the pattern language. A $\forall$-automaton is a nondeterministic automaton for which accepted sequences have to be accepted in all possible runs. In traditional nondeterministic $\exists$-automata, an accepted sequence only has to be accepted in one possible run. Patterns are automatically translated to automata. The automaton language forms a subset of the powerful rule-based RULER specification language [1-4], with some modifications. The automaton language has been designed to be sufficiently expressive to support the actual encountered needs of the MSL testing engineers, and yet as simple as possible to minimize learning effort. RULER is a much more comprehensive specification language, resembling a functional stream programming language. The differences between LOGSCOPE and RULER are explained at the end of this subsection.

In LOGSCOPE, an automaton is expressed in terms of states and transitions between states triggered by events. Events are exactly as defined for patterns. Just as events can be parameterized with values, so can states be parameterized, carrying values produced by incoming transitions. Automata, handwritten as well as translated from patterns, can be visualized with GRAPHVIZ [5]. Automata are stored in GraphViz's dot format for diagrams. Automata visualization has proved useful for users of the pattern language when trying to ensure that their patterns express what is intended; we believe that textual development with graphic visualization is a very effective method for experienced test engineers. We illustrate the automaton language by presenting the automata for patterns P3 and $\mathrm{P} 4$.

\section{A. Automaton for Pattern P3}

The automaton corresponding to pattern P3 is shown in Fig. 4 and visualized in Fig. 5. The automaton consists of four states: $\mathrm{S} 1, \mathrm{~S} 2, \mathrm{~S} 3$, and $\mathrm{S} 4$, where the first mentioned state is the initial state (can also be identified via an initial keyword, as there may be several initial states). There is one transition exiting S1: this transition is triggered by a flight software command, binding $\mathrm{x}$ and $\mathrm{y}$, and entering state $\mathrm{S} 2(\mathrm{x}, \mathrm{y})$ with $\mathrm{x}$ and $\mathrm{y}$ now bound to the actual values in the matching event - an example of a state parameterized with data. The S1 state is an always-state, meaning that it is always active, waiting for any command observed. As such several instances of $S 2(x, y)$ can be active at any point in time, each binding different values to $\mathrm{x}$ and $\mathrm{y}$.

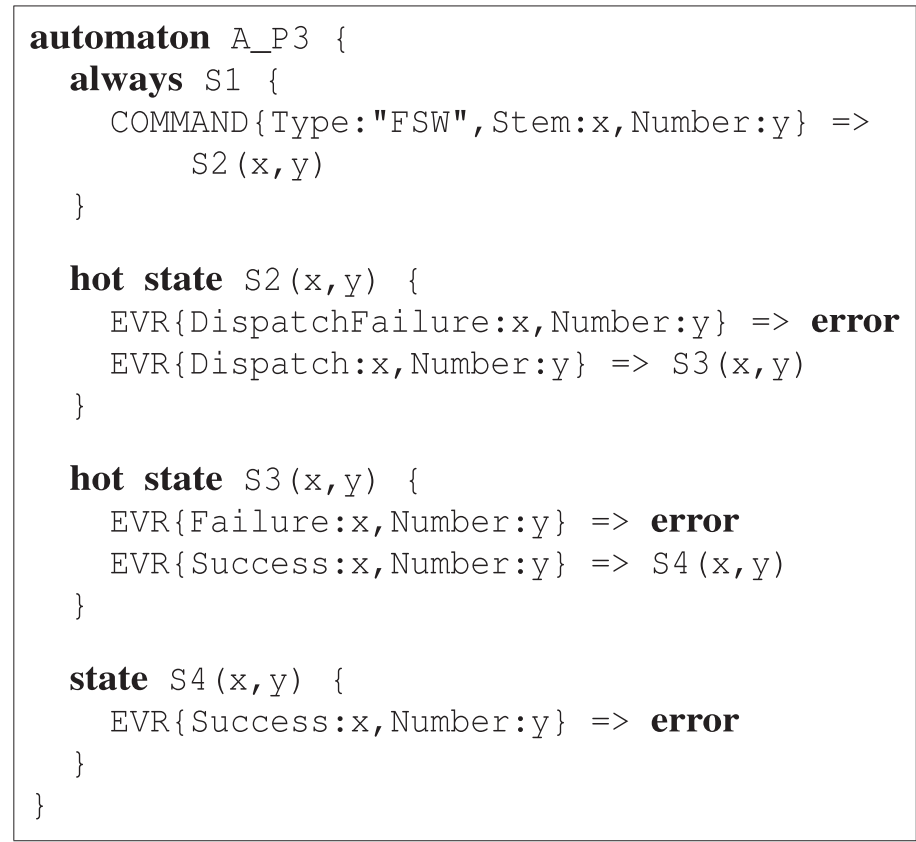

Fig. 4 The automaton A_P3 for property P3. 


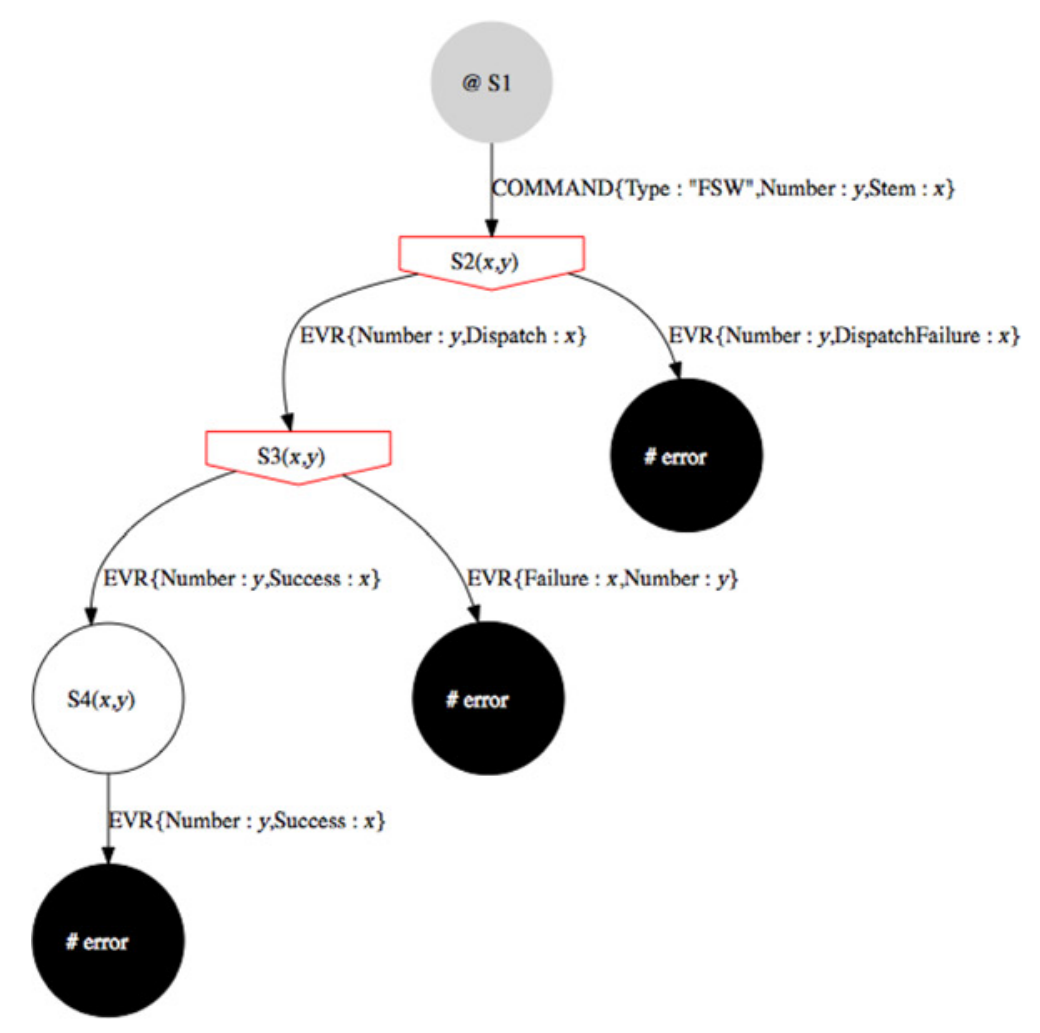

Fig. 5 Visualization of automaton A_P3.

States S2 and S3 are so-called hot states, meaning that instances of these states should be left (disappear) before the end of the log is analyzed; if not, it is regarded as an error. They are used to model that some event must occur before the end of the log. It is also possible to declare states hot with a separate hot-state declaration, as in: "hot S2, S3". In this case, state S2, for example, represents the property that a dispatch should occur (with no previous dispatch failure). The first exiting transition is matched by any EVR with a DispatchFailure field with a value equal to the parameter $\mathrm{x}$ and a Number field with a value equal to the parameter $\mathrm{y}$; and similarly for the second transition. There are two special states: the error state (indicating error) and the done state (indicating successful termination of a branch of the automaton). The state $S 4$ is a normal state (not an always-state and not a hot-state), meaning that it is acceptable to finish monitoring in that state. Its purpose is to check that there are no further successes after the first, for a particular command $\mathrm{x}$ with number $\mathrm{y}$.

Automaton visualization uses different symbols for the different states: a gray state represents an initial state, a white circle represents a normal state, a black circle represents an error state, and a downward-pointed pentagon represents a hot state. The "@" symbol in state S1 indicates an always-state.

\section{B. Automaton for Pattern P4}

The pattern P4 is translated into the automaton in Fig. 6 and visualized in Fig. 7. This automaton expresses that after a command, we want to see a dispatch (state S2) and a success (state S3), and after that no further success (state $S 6$ ), but in no particular order, and that there should not at any time be a dispatch failure (state $S 4$ ) or a failure (state S5). The transition of state S1 enumerates several target states, all of which become active when the transition fires, and all of which must lead to success: no error states should be entered and at the end no hot states should be active. The states can be said to "execute in parallel". The visualization of this automaton uses an upward-pointing triangle (similar to a " $\wedge$ ") to describe a transition with multiple targets. Note that done is visualized as a white circle (a normal state) labeled \#done. 


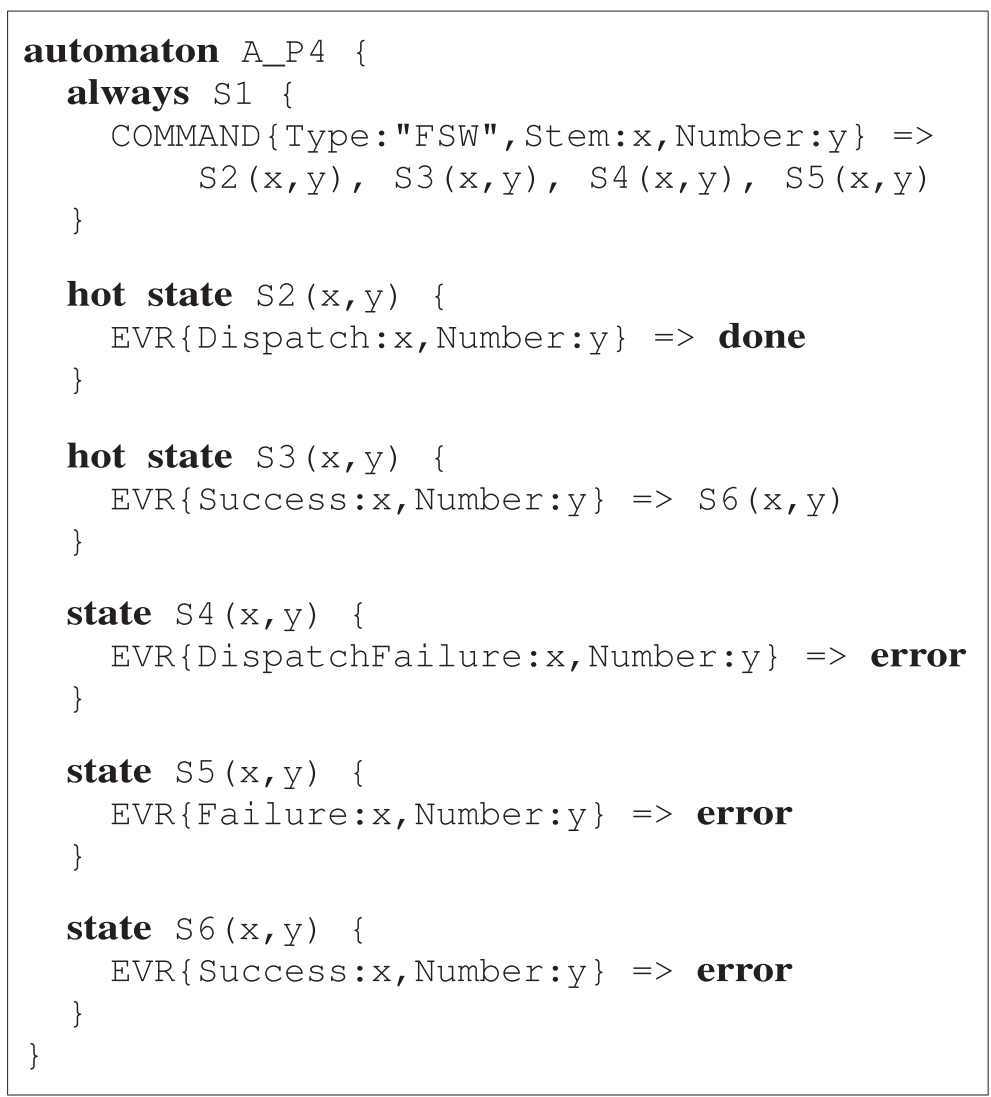

Fig. 6 The automaton A_P4 for property P4.

\section{Other Language Concepts}

The specification language offers a number of additional features. These include a notion of success states, a dual to hot states. Occasionally, it can be more convenient to express a property using success states: at least one of these must be reached by the end of the log analysis. States can also be defined as step states, meaning that in case the next event does not trigger one of the transitions, monitoring along that path is terminated. This is not an error, unless it means that some success state is not reached. This concept is specifically used during learning.

The language supports both C-style multiline comments (/* . . */) and PYTHON-style single-line trailing comments (\# . . .). Furthermore, it is possible to ignore a pattern or automaton by prefixing the declaration with the keyword ignore. This is useful for switching specifications on and off, as an alternative to commenting them out.

As mentioned earlier, the automaton language is more expressive than the pattern language. Recall that a pattern has the form: event $=>$ consequence; that is, the antecedent is a single event. It is therefore currently not possible to write a pattern, for example, of the form: $\left[e_{1}, e_{2}, e_{3}\right]=>e_{4}$, with the intended meaning that if events $e_{1}, e_{2}$, and $e_{3}$ occur, then $e_{4}$ should occur, despite this property being easily expressed in the automaton language. The pattern language can, of course, be extended to also allow this form of property (work in-progress).

\section{Relationship to RULER}

As mentioned above, the LOGSCOPE automaton language forms a simplified subset of the RULER language needed for translating the pattern language. As such it represents an interesting simple but powerful language, focusing on data parameterization, for writing monitors. The RULER concepts that have been ignored are the following. Whereas LOGSCOPE supports state machines where a transition in one state is triggered by one event and leads to a conjunction of resulting states, RULER is indeed a rule-based system, where a transition can be triggered by a conjunction of 


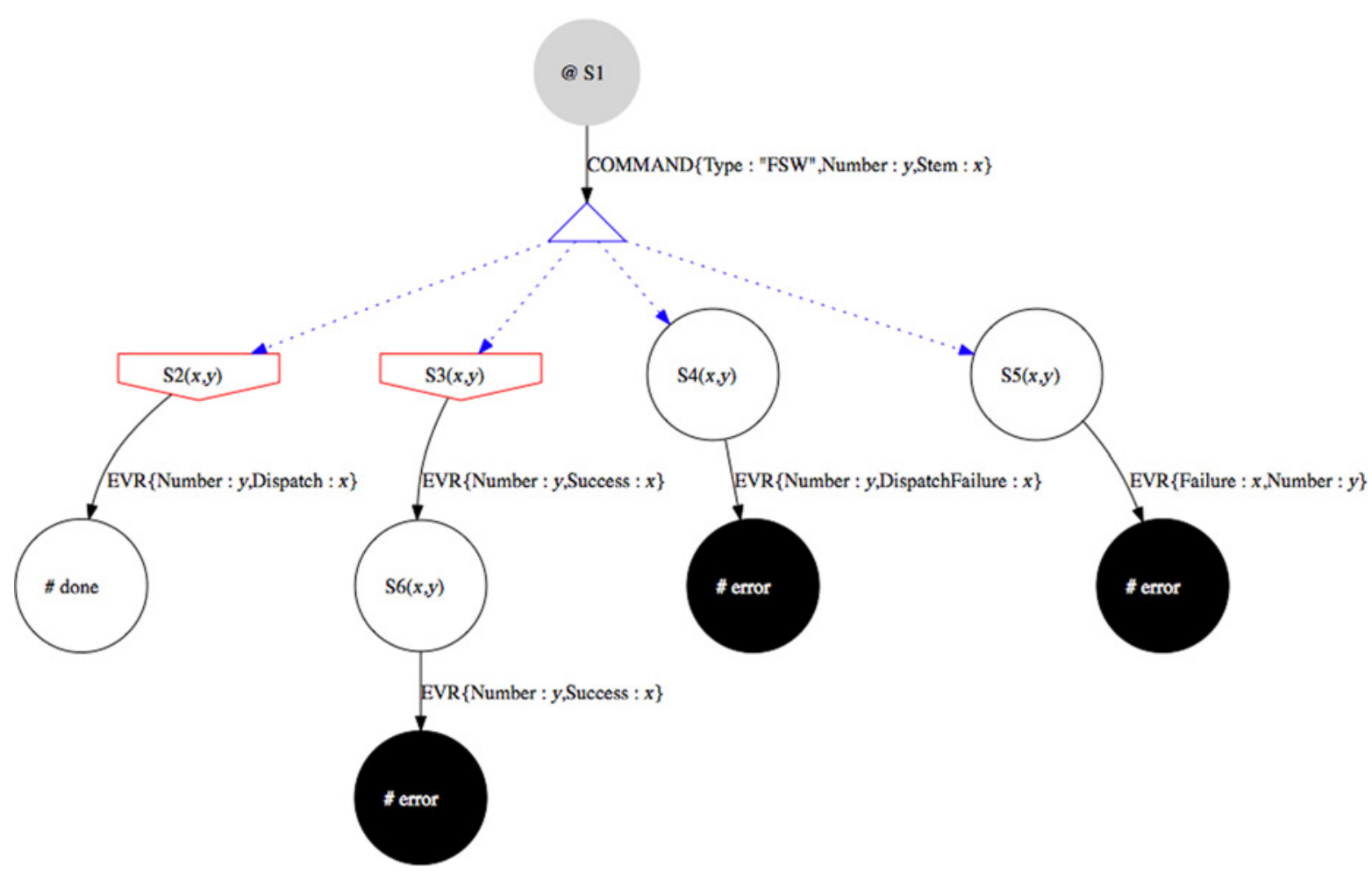

Fig. 7 Visualization of automaton A_P4.

states, events, and negations of such; and where the result is a disjunction of conjunctions of states and negations of such (negating a state means removing it). In RULER disjunction is used to represent alternative ways of achieving success. Note that what is referred to as a state in LOGSCOPE is referred to as a rule activation in RULER. RULER in addition has a notion of super state, similar to that in statecharts/UML. In RULER, templates (which correspond to LOGSCOPE automata) can themselves be parameterized with data as well as with other templates, making RULER higher order. Furthermore, various combinators exist for composing templates (sequential compositon, conditionals, parallel composition, etc.).

\section{E. Type Checking}

In addition to the types of events, we must consider the types of event fields. User specifications are statically type-checked to ensure consistencies within a specification; we omit the details of this very standard (and, in this case, fairly trivial) process. Unfortunately, static checking does not detect the most common and serious type errors: inconsistencies between a specification and a $\log$. Because we provide a very flexible specification language, agnostic as to the deep structure of events, we must handle these dynamic type errors at run-time, when we can compare with a log. Dynamic type errors can be divided into two classes: field errors and range errors. In field errors, a field is mentioned in the specification but is not present in the log, often due to misspellings or (less commonly) undocumented changes to the log database structure by the MSL ground system or flight software. Range errors generally concern a case where the specification expects one type (i.e., a string, integer, or list) and the log provides a different type object for the field. Another form of range error is when a specification indexes an event field, but the index is out-of-bounds (negative or larger than the size of the object). Our (somewhat ad hoc) solution is to provide users with a list of all patterns and automata that never trigger (which may indicate an error in a field's name, or simply an event that did not occur) and all range violations after checking a specification. To improve usability (and in the spirit of PутноN) we also provide limited automatic conversions (e.g., of strings containing numbers to numbers) while still reporting the range error in question. 


\section{Learning}

Writing specifications can be difficult and time consuming. The key problem consists in identifying what properties to check - even formulated in English prose. An approach is to generate logs during one or more runs, and then look at (eyeball) the logs while trying to extract correct-behavior patterns. This process can be supported by tools in various ways. One extreme approach is to attempt to learn automatically a specification from one or more presumably "well behaved" logs, and then later turn this specification into a monitor to check subsequent logs as part of a regression test suite. If these differ, for example, due to later code modifications, warnings highlighting the discrepancies are issued. This approach assumes that a user can judge whether an initial set of logs (those to be learned from) reflect correct program behavior, and is, of course not a generally sound approach, especially if logs contain thousands of events. If the initial logs are the result of erroneous program behavior, the learner will learn a wrong specification. However, as long as error reports are considered with care, this technique is effective for catching bugs and "characterizing" software that is in a constant state of flux.

LOGSCOPE can learn exact logs it observes up to equivalence on a set of field names that is provided to the learner, either from a default set or a set (for each kind of event) provided by the user. Two events are considered equivalent if they are equal with respect to these fields. The learner module, when given several logs, will build an automaton that represents the set of all logs seen. Common prefixes of the logs will result in a single path through the automaton, which will eventually form a tree-shape (no loops). The leaf-states are success states (at least one success state must be reached before the end of the log), and the remaining states are step states (states that must be left in the next step, hence forcing complete conformance to the automaton; no extra events are allowed).

It is possible to create a new automaton, learn from one or more logs, write back the automaton learned so far to persistent memory, and then later further refine it via learning or use it for monitoring. This form of learning results in typically very large automata. The objective is in this case similar to that of comparing logs with UNIX's diff command. Here one would possibly also attempt to remove irrelevant event fields, for example, with UNIX's grep command. Using an automata-based approach, however, opens up the possibility of more advanced learning of logs, what we term abstract learning.

The learning facility has not been fully applied but addresses a need by test engineers to i) run a set of nominal test runs in their office, ii) check by hand that the generated logs conform to expectations, and iii) at a later stage go to a software/hardware testing laboratory and rerun the tests, this time automatically checking that the results match those of previous runs.

\section{A. Running LOGSCOPE}

\section{Usage and Case Study}

LOGSCOPE is called from a PYTHON application as in the following script, which first creates a log (typically by reading in a log generated by a running program), then creates an instance of an Observer object (given the location of the specification(s), possibly in a list, as parameter), and then applies the moni tor method to the log as follows:

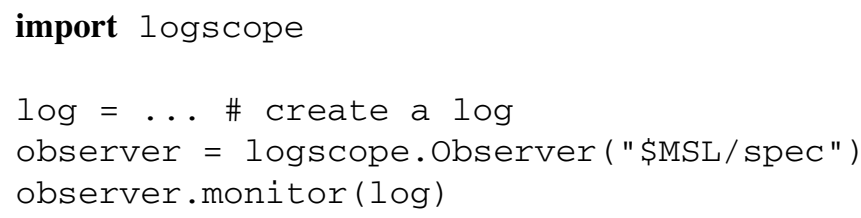

Executing the above PYTHON script will generate a file with results as well as a set of GraphViz dot-files visualizing the generated automata. The results of the monitoring can also be accessed from within the PYTHON script by a getResults ( ) method, so that they can be processed, e.g., as part of a regression test harness.

Our original $\log$ in Fig. 2 violates some of the properties presented in this paper. This is summarized by the system as follows: 


\section{BARRINGER ET AL}

\begin{tabular}{|c|c|c|c|}
\hline \multicolumn{4}{|c|}{$\begin{array}{c}\text { Summary of Errors: } \\
======================\end{array}$} \\
\hline P1 & : & 1 & error \\
\hline P2 & : & 0 & \\
\hline P3 & : & 1 & error \\
\hline $\mathrm{P} 4$ & : & 3 & errors \\
\hline $\mathrm{P} 5$ & : & 0 & \\
\hline P6 & : & 0 & \\
\hline $\mathrm{P} 7$ & : & 0 & \\
\hline P8 & : & 0 & \\
\hline P9 & : & 3 & errors \\
\hline A_P3 & : & 1 & error \\
\hline A_P4 & : & 3 & errors \\
\hline
\end{tabular}

All these violations are caused by the dispatch failure (and subsequent lack of a dispatch and a success) of the last command issued (event number 9626). To simplify this presentation, we shall focus on property A_P4 presented in Sec. V.B and visualized in Fig. 7. This property is similar to P4. A_P4 is violated three times: once because a failure occurs, and twice because neither a dispatch nor a success occurs. The error messages for the dispatch failure and the lack of dispatch are listed below.

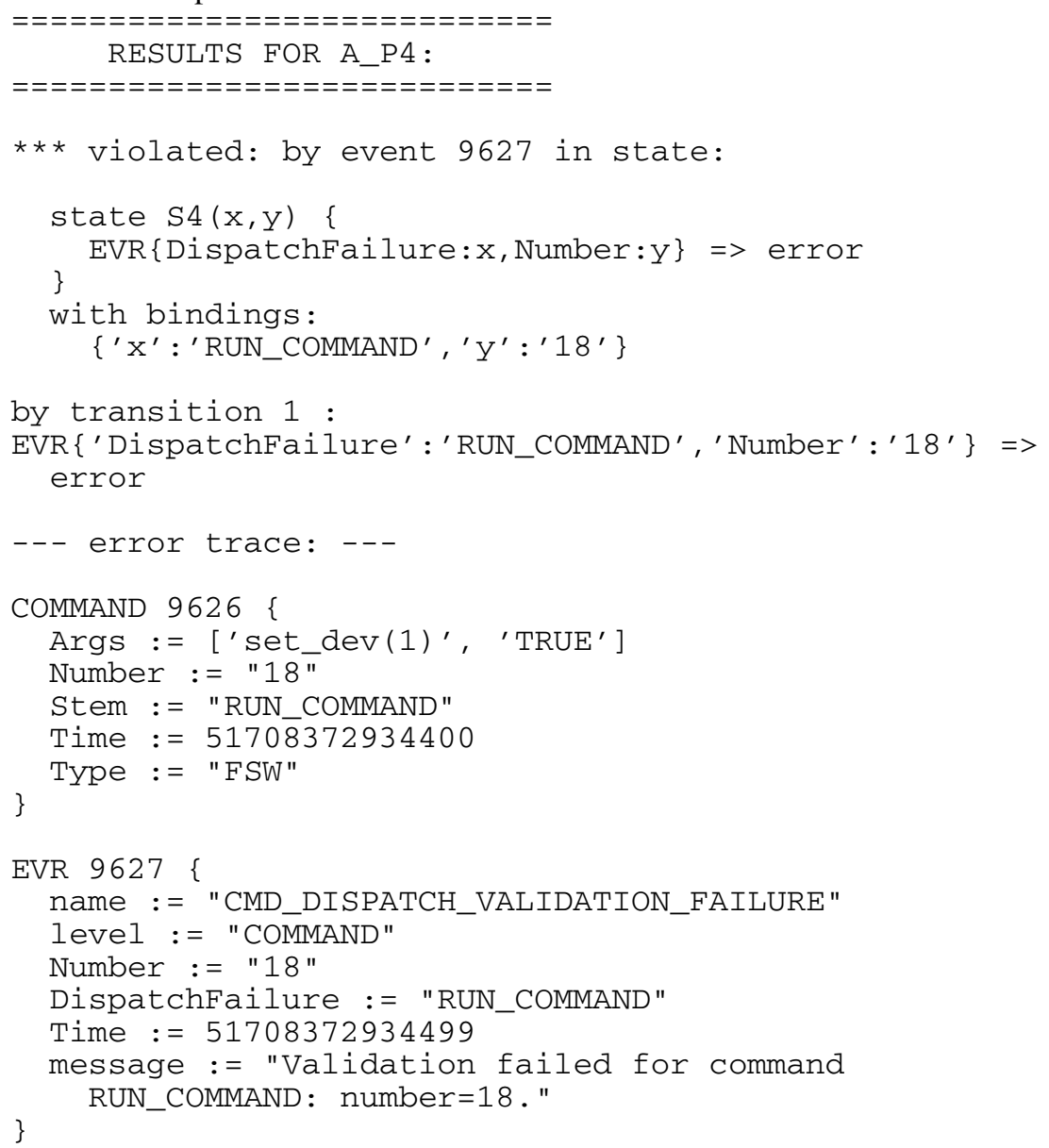




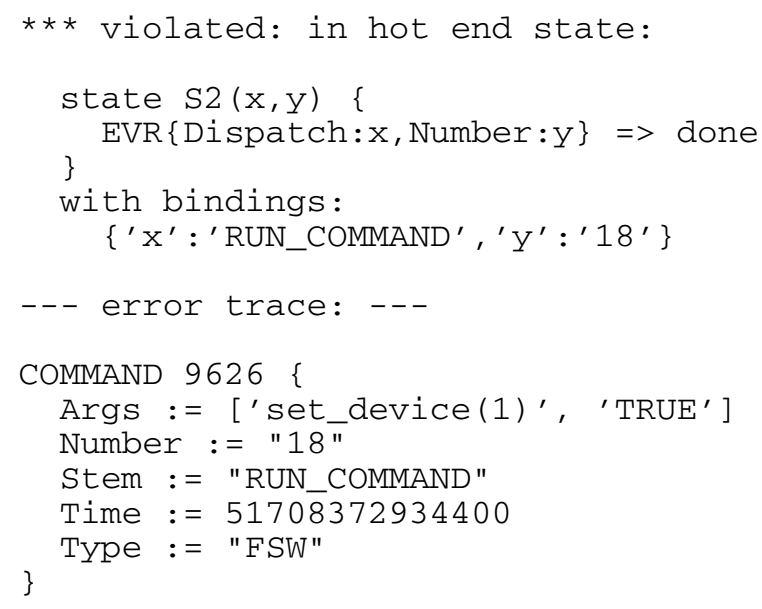

The first error message explains the violation that the dispatch failure event number 9627 triggers the error transition in the state $\mathrm{S} 4$, which is listed, including the values that are bound to the state parameters $\mathrm{x}$ and $\mathrm{y}$. The error trace only contains the events that have made the automaton move: the RUN_COMMAND command event and the fatal dispatch failure event for that command. Since events are numbered, it is easy to locate these events in the real $\log$, which is also stored with event numbers.

The second error message explains the lack of a dispatch. The hot state S2 is where the monitor rests at the end of the log, indicating a violation. The only event that moved the monitor with respect to this particular violation is the initial RUN_COMMAND command, which is the only event listed in the error trace.

\section{B. Application in MSL Testing}

As described, our tool and language development was guided generally by the needs of the MSL test engineers. Our first trial run, initiated at the request of MSL software management, checked the core behavior of the command dispatch and success protocol shown above as pattern P3, for 400 issued commands (an automated regression test predating our tool). Using LOGSCOPE enabled us to more easily experiment with the specification compared to what a test engineer could do using PYTHON. This led to the discovery of a previously uncaptured error, namely, duplicated success EVRs. A more extensive application, initiated by the test engineer responsible, was the automatic generation of specifications from tests of the power module. The test engineer replaced previously unreliable on-the-fly queries to the ground software with code to generate a specification for each tested behavior. Enabling this automatic generation was a primary motivation for some new language features, including pattern scopes. Again, log analysis revealed several faulty behaviors in the power and command modules, and revealed subtle issues with the timing of channel telemetry. The sample log in Fig. 2 is taken from a power test (the full log contains over 11,000 events, including 107 flight software commands). The test engineers responsible for the file verification system (used when the ground sends files via an uplink to the spacecraft) and the PYRO system (used to fire pyros) also replaced hand-scripted test code with LOGSCOPE specifications. In the case of the PYRO module, the test engineer needed the capability to learn a canonical log and compare other logs against the known correct result, a primary motivation for the learning capabilities of the tool.

We believe that developing the tool in collaboration with the test engineers (and the larger MSL flight software team) has maximized the tool's adoption and utility, and can serve as a model for the introduction of formal specification methods in other software efforts. Indeed, it seems clear that the tool improved the test team's productivity and could result in better-tested flight software.

\section{Conclusions and Future Work}

We have in this paper presented a log analysis specification language offering two forms of specifications: temporal logic patterns and $\forall$-automata, both of which can be parameterized with data, primitive and composite (lists, maps). 
The automaton language forms conceptually a subset of the RULER language, limited to the concepts needed by MSL. In this sense, the automaton language presented here suggests a useful, and yet simple subset of RULER. The automaton language is more expressive than the pattern language and can be used in cases where this extra expressiveness is required. Data-parameterized patterns are automatically translated to data-parameterized automata. A point of particular interest is that engineers find it very effective to write specifications in the pattern language and check their precise semantics observing the automaton visualizations. The pattern language itself is new and interesting in the sense that engineers are able to learn it fairly quickly, and they found that it was useful for expressing most of the realistic properties.

The decision to analyze logs probably turned out to be crucial to the adoption of formal specifications by a testing team at JPL. Logs were already produced by the system under test, and hence no instrumentation effort on behalf of the flight software engineers was required (and, therefore, no problem with breaking expected real-time performance). This meant that the testing team could work more or less in isolation, without slowing the flight software engineers down. A great amount of research in runtime verification involves instrumentation issues. Automated code instrumentation is indeed important and interesting. However, it might not be a simple matter for software engineers to decide what to monitor and how to specify it. For example, specifying pointcuts in aspect-oriented programming [37] can be a challenge. On the other hand, inserting print statements in code is the world's most common method for debugging and understanding programs [38]. Our recommendation is, therefore, to apply formal runtime verification to logs when automated code instrumentation becomes impractical. Even unstructured logs can be made useful, though structured logging, with events as records, is generally preferable. We experimented with extracting events from text logs, using regular expressions to define events, e.g., in this example where command is bound to the number matched by $(\backslash d+)$ :

event success (command) $=$

'COMMAND $((\backslash d+)) \backslash(. * ? \backslash)$ SUCCEEDED'

We also hope to establish a connection between requirements engineering and logs, such that requirements become testable through runtime verification of logs. The common element is the event: requirements should be expressed as predicates on sequences of events, and logging should produce those events. The reactions we received during MSL testing suggest that event-sequences may be a very natural way for systems engineers (not limited to software engineers) working on requirements to formulate properties.

Further future work includes merging a more expressive version of the LOGSCOPE temporal logic with the RULER system [2]. A merge should, however, diminish the distinction between temporal logic and rules (rather than translating one into the other), while still providing the benefits of both. Another line of work is to explore learning techniques. The learning techniques implemented are rather simple (just learning the traces seen, abstracted to certain events and fields). It would be desirable to learn high-level properties about consequences of individual commands, yielding typically small, human readable specification units, an idea similar to the approach of Perracotta [39]. For example, for each command one can learn what subsequent events always occur between the command and the next command. This corresponds to introducing a scope for learning the consequences of a single command. One can learn the specific order in which subsequent events occur or ignore the order. One can learn minimal and maximal time periods in between events. We also hope to incorporate classic automata learning results [40,41], though the challenge here is considerable: the language of input symbols is very large, and it is not feasible, for example, to make precise language-inclusion queries as required by Angluin's algorithm. A final goal is to give LOGSCOPE more exposure inside JPL and exploit its potential in other flight missions.

\section{Acknowledgments}

The research described in this paper was carried out at the Jet Propulsion Laboratory, California Institute of Technology, under a contract with the National Aeronautics and Space Administration. Britain's Royal Academy of Engineering, furthermore, provided an award to support this work. We would finally like to thank members of the MSL team: Chris Delp, Dave Hecox, Gerard Holzmann, Rajeev Joshi, Cin-Young Lee, Alex Moncada, Cindy Oda, Glenn Reeves, Lisa Tatge, Hui Ying Wen, Jesse Wright, and Hyejung Yun. 


\section{Appendix: LogScope Grammar}

\section{A. Lexical Elements}

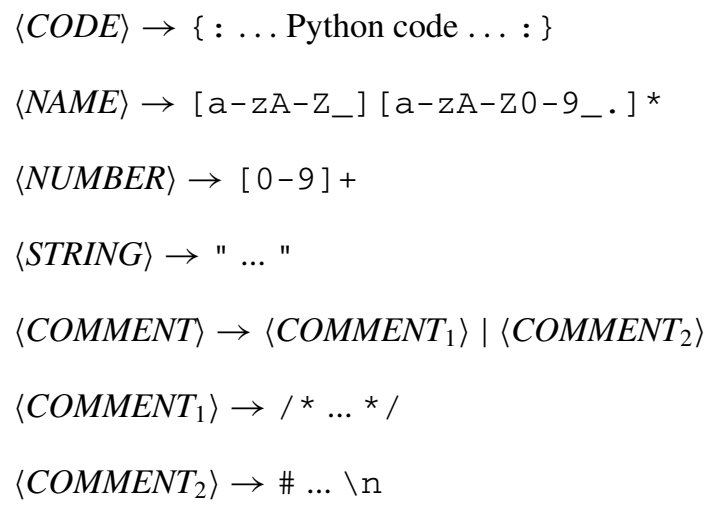

\section{B. Grammar}

1. Specifications

$\langle$ specification $\rangle \rightarrow[\langle C O D E\rangle]\langle\text { monitor }\rangle^{+}$

$\langle$ monitor $\rangle \rightarrow$ [ignore] $\langle$ monitorspec $\rangle$

$\langle$ monitorspec $\rangle \rightarrow\langle$ pattern $\rangle \mid\langle$ automaton $\rangle$

\section{Patterns}

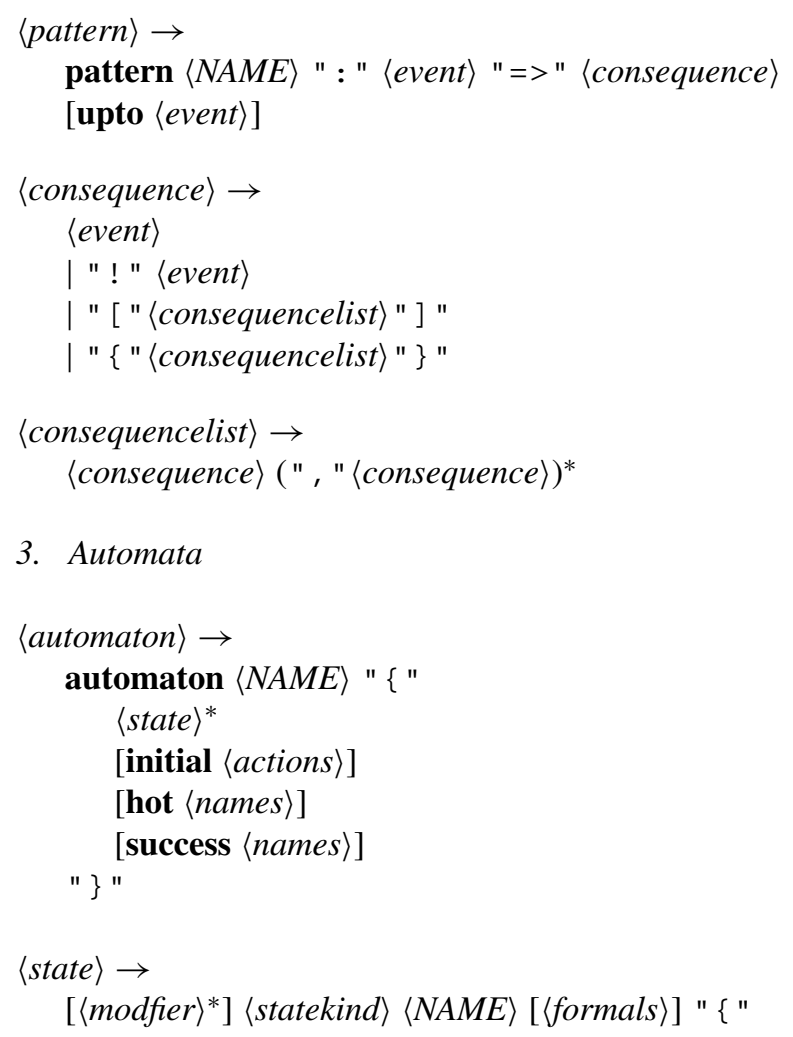




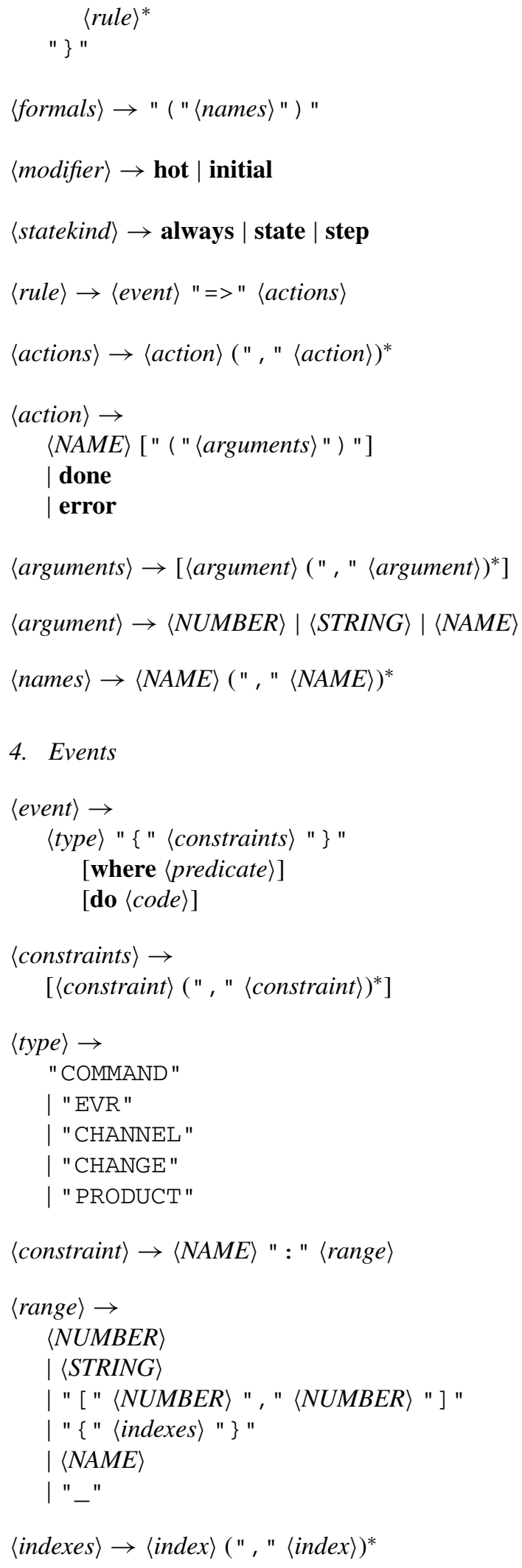




\section{BARRINGER ET AL.}

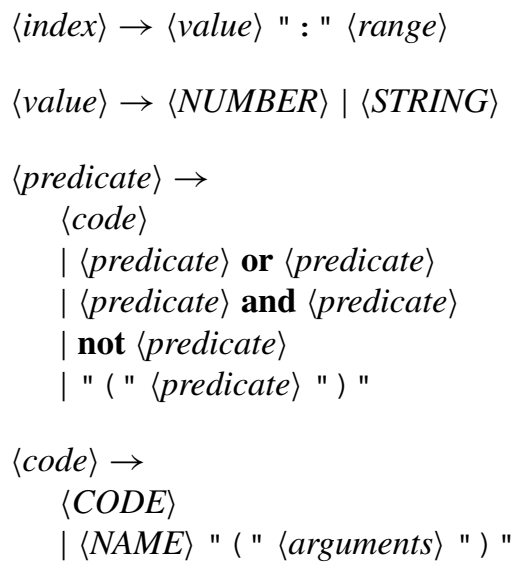

\section{References}

[1] Barringer, H., Havelund, K., Rydeheard, D., and Groce, A., "Rule Systems for Runtime Verification: A Short Tutorial," edited by S. Bensalem, and D. Peled, Proceedings of the 9th International Workshop on Runtime Verification (RV'09), LNCS 5779, Springer, Berlin, Germany, 2009, pp. 1-24.

[2] Barringer, H., Rydeheard, D., and Havelund, K., "Rule Systems for Run-Time Monitoring: From Eagle to RuleR," Proceedings of the 7th International Workshop on Runtime Verification (RV'07), Vancouver, Canada, LNCS 4839, Springer, Berlin, Germany, 2007, pp. 111-125.

[3] Barringer, H., Rydeheard, D., and Havelund, K., "Rule Systems for Run-Time Monitoring: from Eagle to RuleR," Journal of Logic and Computation, Vol. 20, No. 3, 2010, pp. 675-706. doi: 10.1093/logcom/exn076

[4] Barringer, H., Rydeheard, D. E., and Havelund, K., "RuleR: A Tutorial Guide," http://www.cs.man.ac.uk/ howard/LPA.html, 2008.

[5] GraphViz: http://www.graphviz.org.

[6] http://mars.jpl.nasa.gov/msl.

[7] Smith, M., and Havelund, K., "Requirements Capture with RCAT," Proceedings of the 16th IEEE International Requirements Engineering Conference (RE'08), Barcelona, Spain, IEEE Computer Society, Washington, DC, 2008, pp. 183-192.

[8] Havelund, K., "Runtime Verification of C Programs," Proceedings of the 1st TestCom/FATES Conference, Tokyo, Japan, LNCS 5047, Springer, Berlin, Germany, 2008, pp. 7-22.

[9] Groce, A., Havelund, K., and Smith, M., "From Scripts to Specifications-The Evolution of a Flight Software Testing Effort," Proceedings of the 32nd International Conference on Software Engineering (ICSE'10), 2010, Proceedings, ACM $S I G$, South Africa, Vol. 2, ACM Press, New York, NY, pp. 129-138.

[10] http://www.runtime-verification.org.

[11] Allan, C., Avgustinov, P., Christensen, A. S., Hendren, L., Kuzins, S., Lhoták, O., de Moor, O. Sereni, D., Sittamplan, G., and Tibble, J., "Adding Trace Matching with Free Variables to AspectJ", Proceedings of the 20th Annual ACM SIGPLAN Conference on Object-Oriented Programming, Systems, Languages, and Applications (OOPSLA'05), San Diego, CA, 2005, ACM Press, New York, NY, pp. 345-364.

[12] Barringer, H., Goldberg, A., Havelund, K., and Sen, K., "Rule-Based Runtime Verification," Proceedings of the 5th International VMCAI Conference (VMCAI'04), Venice, Italy, LNCS 2937, Springer, Berlin, Germany, 2004 , pp. 44-57.

[13] Chen, F., and Roşu, G., "MOP: An Efficient and Generic Runtime Verification Framework," Proceedings of the 22nd Annual ACM SIGPLAN Conference on Object-Oriented Programming, Systems, Languages and Applications (OOPSLA'07), Montreal, Canada, 2007, ACM Press, New York, NY, pp. 569-588.

[14] D’Amorim, M., and Havelund, K., "Event-based Runtime Verification of Java Programs," Workshop on Dynamic Program Analysis (WODA'05), 2005, St. Louis, MO, ACM Press, New York, NY, SIGSOFT Softw. Eng. Notes, Vol. 30, No. 4, pp. $1-7$.

[15] Drusinsky, D., “The Temporal Rover and the ATG Rover," Proceedings of the 7th International SPIN Workshop on SPIN Model Checking and Software Verification (SPIN'00), Palo Alto, CA, LNCS 1885, Springer, Berlin, Germany, 2000, pp. 323-330.

http://dx.doi.org/10.1016/j.entcs.2006.02.007 


\section{BARRINGER ET AL.}

[16] Drusinsky, D., "Modeling and Verification using UML Statecharts", Elsevier, Amsterdam, Netherlands, ISBN-13: 978-07506-7949-7, 2006, p. 400.

[17] Eckmann, S., Vigna, G., and Kemmerer, R. A., STATL Definition, Reliable Software Group, Department of Computer Science, University of California, Santa Barbara, CA, 2001.

[18] Falcone, Y., Fernandez, J.-C., and Mounier, L., "Runtime Verification of Safety-Progress Properties", edited by S. Bensalem, and D. Peled, Proceedings of the 9th International Workshop on Runtime Verification (RV'09), Grenoble, France, LNCS 5779, Springer, Berlin, Germany, 2009, pp. 40-59.

[19] Havelund, K., and Roşu, G., "Efficient Monitoring of Safety Properties," Software Tools for Technology Transfer, Vol. 6 No. 2, 2004, pp. 158-173.

[20] Kim, M., Kannan, S., Lee, I., and Sokolsky, O., "Java-MaC: a Run-time Assurance Tool for Java," Proceedings of the 1st International Workshop on Runtime Verification (RV'01), Paris, France, ENTCS 55(2), Elsevier, Amsterdam, Netherlands, 2001, pp. 218-235.

[21] Stolz, V., and Bodden, E. “Temporal Assertions using AspectJ," Proceedings of the 5th International Workshop on Runtime Verification (RV'05), ENTCS, Edinburgh, Scotland, UK, Elsevier, Amsterdam, Netherlands, Vol. 144, No. 4, 2006, pp. 109-124.

http://dx.doi.org/10.1016/j.entcs.2006.02.007

[22] Kiczales, G., Hilsdale, E., Hugunin, J., Kersten, M., Palm, J., and Griswold, W. G., “An Overview of AspectJ,” edited by J. Lindskov Knudsen, 15th European Conference on Object-oriented Programming, Budapest, Hungary, LNCS 2072, Springer, Berlin, Germany, 2001, pp. 327-353.

[23] Bodden, E., "J-LO-A Tool for Runtime-Checking Temporal Assertions," Master's Thesis, RWTH Aachen University, 2005.

[24] Andrews, J. H., and Zhang, Y., "General Test Result Checking with Log File Analysis," IEEE Transactions on Software Engineering, Vol. 29, No. 7, 2003, pp. 634-648. http://dx.doi.org/10.1109/TSE.2003.1214327

[25] Chang, F., and Ren, J., "Validating System Properties Exhibited in Execution Traces," Proceedings of the 22nd IEEE/ACM International Conference on Automated Software Engineering (ASE'07), Atlanta, GA, 2007, ACM Press, New York, NY, pp. 517-520.

[26] Vardi, M., From Church and Prior to PSL, 25 Years of Model Checking: History, Achievements, Perspectives, Springer, Berlin, Germany, LNCS 5000, 2008, pp. 150-171.

[27] Bauer, A., Leucker, M., and Streit, J., "SALT—Structured Assertion Language for Temporal Logic," Proceedings of the 8th International Conference on Formal Engineering Methods (ICFEM'06), Macao, China, 2006, LNCS 4260, Springer, Berlin, Germany, pp. 757-775.

[28] Mateescu, R., and Thivolle, D., "A Model Checking Language for Concurrent Value-Passing Systems," Proceedings of the 15th International Symposium on Formal Methods (FM'08), Turku, Finland, LNCS 5014, Springer, Berlin, Germany, 2008, pp. 148-164.

[29] Coen-Porisini, A., Denaro, G., Ghezzi, C., and Pezzè, M., "Using Symbolic Execution for Verifying Safety-Critical Systems," ESEC/FSE-9: Proceedings of the 8th European Software Engineering Conference, Vienna, Austria, ACM Press, New York, NY, 2001, pp. 142-151.

[30] Dillon, L. K., Kutty, G., Moser, L. E., Melliar-Smith, P. M., and Ramakrishna, Y. S., "A Graphical Interval Logic for Specifying Concurrent Systems," ACM Transactions on Software Engineering and Methodology, Vol. 3, No, 2, 1994, pp. 131-165. http://dx.doi.org/10.1145/192218.192226

[31] Dillon, L. K., and Ramakrishna, Y. S., "Generating Oracles from Your Favorite Temporal Logic Specifications," $A C M$ SIGSOFT Software Engineering Notes Archive, Vol. 21, No. 6, 1996, pp. 106-117. http://dx.doi.org/10.1145/250707.239116

[32] Alavi, H., Avrunin, G., Corbett, J., Dillon, L., Dwyer, M. and Pasareanu, C., Specification Patterns, SAnToS Laboratory, Kansas State University, http://patterns.projects.cis.ksu.edu.

[33] Kortenkamp, D., Milam, T., Simmons, R., and Fern, J. L., "Collecting and Analyzing Data from Distributed Control Programs," Proceedings of the 1st International Workshop on Runtime Verification (RV'01), Paris, France, ENTCS 55(2), Elsevier, Amsterdam, Netherlands, 2001, pp. 133-151.

[34] Pnueli, A. "The Temporal Logic of Programs," Proceedings of the 18th IEEE Symposium on Foundations of Computer Science (FOCS'77), Rhode Island, IEEE, Washington, DC, 1977, pp. 46-57.

[35] Smith, M., Holzmann, G., and Ettessami, K., "Events and Constraints: A Graphical Editor for Capturing Logic Properties of Programs," Proceedings of the 5th IEEE International Symposium on Requirements Engineering (RE'01), Toronto, Canada, 2001, 55(2), IEEE Computer Society, Washington, DC, pp. 14-22. 


\section{BARRINGER ET AL.}

[36] Barringer, H., Kuiper, R., and Pnueli, A., "Now You May Compose Temporal Logic Specifications," Proceedings of the 16th Annual ACM Symposium on Theory of Computing (STOC'84), Washington, DC, 1984, ACM Press, New York, NY, pp. 51-63.

[37] Laddad, R., AspectJ in Action, Manning, Greenwich, CT, 2003.

[38] Zeller, A., Why Programs Fail: A Guide to Systematic Debugging, Morgan Kaufmann, Burlington, MA, 2005.

[39] Yang, J., Evans, D., Bhardwaj, D., Bhat, T., and Das, M., "Perracotta: Mining Temporal API Rules from Imperfect Traces," Proceedings of the 28th International Conference on Software Engineering (ICSE'06), Shanghai, China, 2006, ACM Press, New York, NY, pp. 282-291.

[40] Angluin, D. "Learning Regular Sets from Queries and Counterexamples," Information and Computation, Vol. 75, No. 2, 1987, pp. 87-106. http://dx.doi.org/10.1016/0890-5401(87)90052-6

[41] Biermann, A. W., and Feldman, J. A., "On the Synthesis of Finite-State Machines from Samples of their Behaviour," IEEE Transactions on Computers, Vol. 21, 1972, pp. 592-597.

Michael Hinchey Associate Editor 